Alberta-Thy 03-98

\title{
Thermal Fields, Entropy, and Black Holes
}

\author{
V.P. Frolov ${ }^{1,2,3}$ and D.V. Fursaev ${ }^{1,4}$ \\ 1 Theoretical Physics Institute, Department of Physics, University of Alberta, \\ Edmonton, Canada T6G 2J1 \\ ${ }^{2}$ CIAR Cosmology and Gravity Program \\ ${ }^{3}$ P.N.Lebedev Physics Institute, Leninskii Prospect 53, Moscow 117924, Russia \\ ${ }^{4}$ Joint Institute for Nuclear Research, Laboratory of Theoretical Physics, \\ 141980 Dubna, Russia \\ e-mails: frolov,dfursaev@phys.ualberta.ca
}

\begin{abstract}
In this review we describe statistical mechanics of quantum systems in the presence of a Killing horizon and compare statistical-mechanical and one-loop contributions to black hole entropy. Studying these questions was motivated by attempts to explain the entropy of black holes as a statistical-mechanical entropy of quantum fields propagating near the black hole horizon. We provide an introduction to this field of research and review its results. In particular, we discuss the relation between the statistical-mechanical entropy of quantum fields and the Bekenstein-Hawking entropy in the standard scheme with renormalization of gravitational coupling constants and in the theories of induced gravity.
\end{abstract}




\section{Introduction}

Thermodynamics and statistical mechanics of black holes is one of the most exciting and rapidly developing areas of black hole physics. Black holes are known to possess the properties similar to the properties of thermodynamical systems [1]. According to this analogy, a black hole has the entropy $S^{B H}=\frac{1}{4 G} \mathcal{A}$ where $\mathcal{A}$ is the surface area of its horizon and $G$ is the Newton constant?. The quantity $S^{B H}$ was introduced in Refs. [3]- [6] and is known as the Bekenstein-Hawking entropy. The temperature of a black hole is $T_{H}=\frac{\kappa}{2 \pi}$ where $\kappa$ is the surface gravity of the horizon.

In the Einstein theory of general relativity the Bekenstein-Hawking entropy is a pure geometrical quantity. The laws of black hole thermodynamics are derived by making use of the classical Einstein equations and rules of the differential geometry only. If we compare black holes with usual thermodynamical systems an important difference can be easily observed: Black holes are nothing but an empty space with a strong gravitational field while a usual body consists of material constituents (atoms, molecules, etc.). Namely this microscopical structure enables one to explain thermodynamical properties of the body in terms of statistical mechanics of its constituents. Does a black hole have internal degrees of freedom which are responsible for the Bekenstein-Hawking entropy? This is a key question of black hole physics.

The black hole entropy problem is important. The statistical-mechanical derivation of the Bekenstein-Hawking entropy is a highly non-trivial test for a fundamental theory of quantum gravity. Recent successful calculations of $S^{B H}$ for extremal [7]-[9] and near extremal [10, [11] black holes in the superstring theory clearly demonstrate this. A review of these computations and further references can be found, for instance, in [12], [13]. Besides the superstring approach there are other approaches which attack the problem of black hole entropy from different directions. The explanation of the entropy of 3D black holes suggested by Carlip et al. [14, 15] or consideration in the framework of the loop quantum gravity [16] are some of them.

The subject of this review is related to the approach which suggests to explain the black hole entropy in terms of its quantum excitations. This idea was formulated first in [17], 18], 19] and it has stimulated a large number of publications.

The properties of physical vacuum, especially in the presence of gravity, are nontrivial. In the state of vacuum there always exist zero-point fluctuations of physical fields. An observer at rest near a horizon would register these zero-point fluctuations in the form of a thermal atmosphere of a black hole [19]-[25]. Historically the first suggestions to relate the entropy of a black hole to the entropy of its thermal atmosphere were made in works by Thorne and Zurek [17] and by t'Hooft [18]. t'Hooft [18] estimated the thermal entropy by assuming that the red-shifted temperature of the atmosphere is $T_{H}$ and showed that the

\footnotetext{
${ }^{1}$ We use units in which $\hbar=c=k_{B}=1$ ( $k_{B}$ is the Bolzmann constant) and sign conventions of book [2] and, thus, we use the signature $(-,+,+,+)$ for the Lorentzian metric.
} 
entropy is proportional to the horizon area $\mathcal{A}$. To avoid the divergences t'Hooft assumed that fields vanish within some distance near the horizon (the corresponding model was called the "brick wall" model). If this distance is of the order of a Planck lengths the thermal entropy turns out to be comparable to $S^{B H}$.

The reason why the static observer near the black hole sees the vacuum as a mixed state is explained by the loss of the information about a part of the quantum system located inside a the black hole horizon. Bombelli, Koul, Lee and Sorkin [26] and Srednicki [27] have showed that even in a flat space when observations in vacuum are restricted by a part of the system, the entropy is not zero and is proportional to the surface area of the restricted region $\Omega$ (for a recent discussion see [28]). Similar result for the entropy was also established for non-zero spin fields [29] and for the pure sates different from the vacuum [30]. The non-vanishing entropy appears because the "observable" and "non-observable" vacuum fluctuations are entangled (correlated) on the boundary of $\Omega$. By taking into account these properties the authors of Refs. [26], [27] suggested to explain $S^{B H}$ as the entropy of entanglement between quantum fluctuations propagating on the different sides of the horizon.

Frolov and Novikov 31 proposed to relate the black hole entropy to the degrees of freedom corresponding to quantum states in the black hole interior. The density matrix of these degrees of freedom can be obtained by averaging the quantum state of the complete system over the states of fields located outside the black hole. For modes in the close vicinity to the horizon this density matrix is thermal. The particles are created in pairs, and only one of the components can be created outside the horizon. A pair inside the black hole is in a pure state and does not contribute to the entropy. For this reason, the statistical mechanical entropy is connected with entanglement, and it can be written in the form of summation over the modes in the black hole exterior. In other words, this approach incorporates main features of the earlier approaches [17, [18], [26].

A remarkable property of a black hole is that its entaglement entropy, and entropy connected with its thermal atmosphere coincide [32]-35]. In what follows we refer to this quantity as to statistical-mechanical entropy.

Small fluctuations of fields (including the gravitational one) propagating in the black hole background can be related to small deformations of the black hole geometry. This can be explicitly demonstrated in the approach using the no-boundary wavefunction of the black hole [36]. For this reason, counting states of quantum fields is connected with the counting the states of quantum excitations of the black hole.

In the general case, the relation of the statistical-mechanical entropy and the "observable" thermodynamical entropy of the black hole is highly nontrivial [37. The quantum fields near a black hole have an important property. Namely, the density of levels of quantum mechanical Hamiltonians of the fields blows out near the horizon. This results in the divergence of the statistical-mechanical entropy. Susskind and Uglum [38] and Callan and Wilczek [34 suggested that this divergence is related to the ultraviolet one-loop di- 
vergences of the theory and it can be removed by renormalizing the Newtonian coupling constant. This observation was strongly supported by the result of Demers, Lafrance and Myers [39] who showed that the standard Pauli-Villars method regularizes the divergences of the statistical-mechanical entropy on the Reissner-Nordstrom background. Moreover in this regularization, the entropy divergence for a minimally coupled scalar field is completely eliminated by the standard ultraviolet renormalization. The same result for general static black-hole backgrounds was proven by Fursaev and Solodukhin [40]. It was done by using the Euclidean formulation of the theory with conical singularities.

Although the observation made in [38, 34] is very important, there are two problems. First, in order to carry out the renormalization one must introduce an infinite (negative) bare entropy, which has no statistical-mechanical origin. Second, in the presence of nonminimal coupling of a field with the curvature, the divergence of the statistical-mechanical entropy and the ultraviolet divergence are different [39]- 44].

Jacobson 45] pointed out that the first problem can be solved if the Einstein gravity itself appears as a result of quantum effects. A suitable simple example is Sakharov's theory of induced gravity 46, 47]. The models of induced gravity which enable one to check this hypothesis were constructed by Frolov, Fursaev and Zelnikov 48. It was demonstrated that the presence of the non-minimal coupling is necessary in order to get a finite induced Newton constant. Moreover, it makes it possible a consistent derivation of the black hole degeneracy by counting the degrees of freedom of constituents [49, [50].

The idea to relate $S^{B H}$ to quantum excitations of a black hole and the pioneering papers [17], 18], [26], [27], [31] stimulated the study of statistical mechanics of quantum fields in the presence of a Killing horizon. More than hundred papers was written on this subject. Our review of these works has two main aims:

1. To describe the statistical mechanics of quantum fields in the presence of a Killing horizon, its methods and obtained results;

2. To discuss the relation between the statistical mechanical entropy and the observable black hole entropy $S^{B H}$.

As an application of these results, we present the statistical-mechanical derivation of the black hole entropy in the models of induced gravity.

We begin with two formulations of finite-temperature quantum theory on static spacetimes which have been discussed in the literature.

The first is the canonical formulation based on the $(3+1)$-split of the spacetime. It allows one to define the free energy $F^{C}$ of the system in terms of the one particle spectrum. The advantage of the canonical formulation is that statistical-mechanical entropy $S^{C}$ can be derived from $F^{C}$ by the standard rules.

The second is the covariant Euclidean formulation. It starts with the calculation of the one-loop effective action $W$ on the Gibbons-Hawking instanton. The free energy $F^{E}$ 
in this approach is $F^{E}=\beta^{-1} W$, where $\beta$ is the inverse temperature. The covariant Euclidean formulation [2 is especially useful in application to black hole thermodynamics.

These two formulations are logically different, and in the presence of a black hole their comparison is non-trivial. The first part of the review is devoted to this problem. In Section 2 we define $F^{E}$ and $F^{C}$ on spacetimes without horizons and show the equivalence of these functionals. In Section 3 we discuss the features of quantum systems related to the horizon. Section 4 reviews the results of the canonical formulation for the spacetimes with horizons. Special attention is payed to divergences connected with the presence of the horizon and methods of their regularization. The covariant Euclidean formulation in the presence of a horizon is given in Section 5. We discuss ultraviolet one-loop divergences and show that the divergent parts of $F^{E}$ and $F^{C}$ are identical in the covariant regularizations, such as Pauli-Villars and dimensional ones.

In the second part of the review we discuss the relation between statistical-mechanical entropy and the observable thermodynamical entropy of a black hole. In Sections 6,7 we demonstrate how the divergencies of statistical-mechanical entropy are removed by the renormalization of gravitational couplings in the tree-level black hole entropy. The presence of the bare classical entropy make it impossible to give a statistical-mechanical explanation of the black hole entropy in the theories which require the ultraviolet renormalization. This difficulty does not exist in the theories of induced gravity. The mechanism of generation of the Bekenstein-Hawking entropy in such theories is described in Section 8. Our summary and conclusions are represented in Section 9. The Appendix is devoted to interpretation of the Noether charge which appears in the renormalization formula for the entropy because of nonminimal couplings of the fields.

\section{Statistical mechanics of quantum fields in a static gravitational field without horizons}

\subsection{Description of the system}

We begin with discussion of statistical mechanics of quantum fields in a static gravitational field without horizons. This theory has been formulated and investigated in a number of papers starting with pioneering works by Gibbons [51], 52], Gibbons and Perry [53], Dowker and Kennedy [54] in the early seventies. A typical example is a quantum field at finite temperature in the gravitational field of a static non-rotating star. The gravitational field is described by the metric

$$
d s^{2}=g_{00} d t^{2}+g_{a b} d x^{a} d x^{b} \quad, \quad a, b=1,2,3 \quad,
$$

\footnotetext{
2The terms "canonical" and "covariant Euclidean" reflect the form of the presentation of the corresponding partition functions (in form of summing over the energy levels and as a functional integral, respectively). It should be emphasized that there is no standard terminology. We use superscripts $C$ and $E$ to refer to the quantities calculated in the canonical and covariant Euclidean formulations.
} 
where $g_{00}(x)<0$. The metric (2.1) depends only on the spatial coordinates $x^{a}$, and so it admits the Killing field $\zeta=\partial / \partial t$. On the spatial infinity the background is asymptotically flat and, by assumption, a time component of the metric $g_{00}$ tends to -1 . Because metric (2.1) does not depend on time, one can easily define the statistical-mechanical ensembles of different fields on this background. We will be dealing with canonical ensembles characterized by the temperature $T=\beta^{-1}$ measured at asymptotic infinity ${ }^{3}$. The local Tolman temperature measured by an observer at a point $x^{a}$ is $T_{l o c}=\left|g_{00}\right|^{-1 / 2} \beta^{-1}$.

To investigate the cases of Bose and Fermi statistics we will be considering, as an example, free scalar $(\phi)$ and Dirac $(\psi)$ fields. The fields obey the Klein-Gordon and Dirac equations

$$
\begin{gathered}
\left(-\nabla^{\mu} \nabla_{\mu}+m^{2}+\xi R\right) \phi=0, \\
\left(\gamma^{\mu} \nabla_{\mu}+m\right) \psi=0,
\end{gathered}
$$

respectively. Here $R$ is the scalar curvature and $\xi$ is the parameter of the non-minimal coupling. The covariant derivatives $\nabla_{\mu}$ are defined according with the spin of the field. The Dirac $\gamma$-matrices $\gamma^{\mu}=\left(\gamma^{0}, \gamma^{\alpha}\right)$ are defined by the standard relations $\left\{\gamma^{\mu}, \gamma^{\nu}\right\}=2 g^{\mu \nu}$. Note that $\gamma^{0}$ is anti-Hermitean matrix. We define the spinor derivative as $\nabla_{\mu}=\partial_{\mu}+\Gamma_{\mu}$, where $\Gamma_{\mu}=\frac{1}{8}\left[\gamma^{\lambda}, \gamma^{\rho}\right] V_{\rho}^{l} \nabla_{\mu} V_{l \lambda}$ is the connection and $V_{\nu}^{l}$ are the tetrads.

The following scalar products defined for solutions of equations (2.2) and (2.3)

$$
\begin{aligned}
<\phi_{1}, \phi_{2}> & =i \int_{\mathcal{B}} d^{3} x \sqrt{{ }^{(3)} g\left|g_{00}\right|^{-1}}\left(\phi_{1}^{*} \partial_{t} \phi_{2}-\phi_{2} \partial_{t} \phi_{1}^{*}\right) \\
& <\psi_{1}, \psi_{2}>=\int_{\mathcal{B}} d^{3} x \sqrt{{ }^{(3)} g} \psi_{1}^{+} \psi_{2}
\end{aligned}
$$

where ${ }^{(3)} g=\operatorname{det} g_{a b}$, are independent of the choice of the total Cauchy surface $\mathcal{B}$.

\subsection{Canonical formulation and single-particle spectrum}

A canonical ensemble at temperature $\beta^{-1}$ is determined by the partition function

$$
Z^{C}(\beta)=\operatorname{Tr} e^{-\beta: \hat{\mathcal{H}}:}
$$

The operator : $\hat{\mathcal{H}}$ : is the Hamiltonian of the secondary quantized field. It determines a unitary evolution of the quantum field with respect to the Killing time $t$. As usual we use the decomposition of field operators onto positive and negative frequencies with respect to

\footnotetext{
${ }^{3}$ It is well known that an infinite bath of thermal radiation is a gravitationally unstable system. In order to deal with a stable situation one may consider a cavity of a finite size filled with the radiation. To characterize the system one can fix the temperature at the boundary or use the red-shifted temperature at infinity. These remarks are also valid for the case of a black hole being in thermal equilibrium with the radiation. For a non-rotating black hole of mass $M$ the size of the cavity must be less than $3 M$ [55. Speaking about the canonical ensemble we always assume that such a boundary exist. Our main subject is properties of ensembles of quantum fields connected with the presence of the horizon. The presence of external boundaries is not important for this consideration. For this reason we are not discussing them in more details.
} 
the time $t$ in order to define the creation and annihilation operators. The normal ordering in (2.6) is with respect to these operators. For this normal ordering the energy of the state with zero temperature (vacuum) vanishes. If the vacuum energy is non-trivial, definition (2.6) can be easily modified. We will discuss this modification later. The canonical free energy of the system is

$$
F^{C}(\beta)=-\beta^{-1} \ln Z^{C}(\beta)
$$

To proceed with the computation of $F^{C}(\beta)$ it is convenient to rewrite Eq. (2.7) in another equivalent form based on a single-particle spectrum. Let $\omega$ be frequency of a field mode with respect to the Killing time $t$. We call the set of these frequencies the singleparticle spectrum. The spectrum of $\omega$ is uniquely defined by the boundary conditions imposed on the system. If the system is given in a finite region with Dirichlet or other conditions on the boundary the spectrum is discrete. Some frequencies in this case can coincide and we introduce the corresponding degeneracy factor $d(\omega)$. Then Eq. (2.7) can be identically rewritten in the form [56], [57]

$$
F^{C}[\beta]=\eta \beta^{-1} \sum_{\omega} d(\omega) \ln \left(1-\eta e^{-\beta \omega}\right)
$$

The factor $\eta$ is related to the statistics, and it takes values $\eta=1$ and $\eta=-1$ for Bose and Fermi fields, respectively. Equation (2.8) is well-defined: the degeneracies $d(\omega)$ of the three-dimensional elliptic operators grow as $\omega^{2}$ at large $\omega$ but due to the exponential cutoff series (2.8) converges.

When the system has the infinite size the spectrum of $\omega$ is fixed by the appropriate asymptotic conditions. A usual requirement is that fields fall fast enough at the spatial infinity. In this case the spectrum is continuous and the sum in (2.8) has to be replaced by the integral

$$
F^{C}[\beta]=\eta \beta^{-1} \int_{0}^{\infty} d \omega \frac{d n(\omega)}{d \omega} \ln \left(1-\eta e^{-\beta \omega}\right)
$$

The quantity $\frac{d n(\omega)}{d \omega} d \omega$ is the number of levels in the interval $(\omega, \omega+d \omega)$. Equation (2.9) can be obtained from (2.8) in the limit when the interval between the levels shrinks to zero.

\subsection{Single-particle Hamiltonian}

The single-particle spectrum can be found from the wave equations of the fields, Eqs. (2.2), (2.3). On the static space (2.1) these equations can be rewritten in the " $3+1$ " form

$$
\left(\partial_{t}^{2}+H_{s}^{2}\right) \phi=0 \quad, \quad\left(i \partial_{t}-H_{d}\right) \psi=0
$$

where the subscripts $s$ and $d$ are used for scalar fields and Dirac spinors, respectively. $H_{s}^{2}$ and $H_{d}$ are three-dimensional differential operators

$$
H_{s}^{2}=\left|g_{00}\right|\left(-\nabla^{a} \nabla_{a}-w^{a} \nabla_{a}+m^{2}+\xi R\right)
$$




$$
H_{d}=-i \gamma_{0}\left[\gamma^{a}\left(\nabla_{a}+\frac{1}{2} w_{a}\right)+m\right]
$$

The 3-dimensional covariant derivative $\nabla_{a}$ is defined in terms of the metric $g_{a b}$ on the hypersurface of constant time $t=$ const. We denote the constant time surface as $\mathcal{B}$. Operations with the index $a$ are performed with the help of $3 \mathrm{D}$-metric $g_{a b}$ on $\mathcal{B}$, see Eq. (2.1), and $w_{a}=\frac{1}{2} \nabla_{\alpha} \ln \left|g_{00}\right|$ is the three-dimensional part of the acceleration vector $w_{\mu}=\left(0, w_{a}\right)$ of the Killing observer.

The operators $H_{s}$ and $H_{d}$ are quantum-mechanical single-particle Hamiltonians because their eigen-values determine single particle spectra. By substituting the wave functions with the fixed energies $\phi(t, \mathbf{x})=e^{-i \omega t} \phi_{\omega}(\mathbf{x}), \psi(t, \mathbf{x})=e^{-i \omega t} \psi_{\omega}(\mathbf{x})$ into Eqs. (2.10) we arrive at the eigen-value problems

$$
H_{s}^{2} \phi_{\omega}(\mathbf{x})=\omega^{2} \phi_{\omega}(\mathbf{x}) \quad, \quad H_{d}^{2} \psi_{\omega}(\mathbf{x})=\omega^{2} \psi_{\omega}(\mathbf{x})
$$

It is easy to check that $H_{s}$ and $H_{d}$ are Hermitean operators with respect to the following inner products

$$
\begin{gathered}
\left(\phi_{1}, \phi_{2}\right)=\int_{\mathcal{B}} d^{3} x \sqrt{{ }^{(3)} g\left|g_{00}\right|^{-1}} \phi_{1}^{*}(\mathbf{x}) \phi_{2}(\mathbf{x}) \\
\left(\psi_{1}, \psi_{2}\right)=\int_{\mathcal{B}} d^{3} x \sqrt{{ }^{(3)} g} \psi_{1}^{+}(\mathbf{x}) \psi_{2}(\mathbf{x})
\end{gathered}
$$

where $\psi^{+}$denotes a Hermitean conjugated spinor. The form of relations (2.14) and (2.15) follows from inner products (2.4)-(2.5) for four-dimensional fields. The inner products (2.14) and (2.15) are used to normalize the modes.

For the convenience of the computations we will also use another representation of $H_{s}^{2}$ and $H_{d}$ which can be obtained by the following transformation of functions and operators

$$
\begin{aligned}
\bar{\phi}=e^{-\sigma} \phi \quad, \quad \bar{\psi} & =e^{-\frac{3}{2} \sigma} \psi, \\
\bar{H}_{s}^{2}=e^{-\sigma} H_{s}^{2} e^{\sigma} \quad, \quad \bar{H}_{d} & =e^{-\frac{3}{2} \sigma} H_{d} e^{\frac{3}{2} \sigma},
\end{aligned}
$$

where $\sigma=-\frac{1}{2} \ln \left|g_{00}\right|$. The inner products for transformed functions can be obtained from Eqs. (2.14) and (2.15) and they have the universal form

$$
\left(\bar{\Phi}_{1}, \bar{\Phi}_{2}\right)=\int_{\mathcal{B}} \sqrt{{ }^{(3)} \bar{g}} d^{3} x\left(\bar{\Phi}_{1}\right)^{+} \bar{\Phi}_{2}
$$

Here $\bar{\Phi}$ denotes either scalar or spinor field, ${ }^{(3)} \bar{g}=\operatorname{det} \bar{g}_{a b}$, and $\bar{g}_{a b}=g_{a b} /\left|g_{00}\right|=e^{2 \sigma} g_{a b}$. Let us stress that transformations (2.16) and (2.17) do not change the spectra determined by Eqs. (2.13). Thus, the operators $\bar{H}_{i}$ and $H_{i}(i=s, d)$ are equivalent.

From Eqs. (2.17) one finds the spinor Hamiltonian

$$
\bar{H}_{d}=i \bar{\gamma}_{0}\left(\bar{\gamma}^{a} \bar{\nabla}_{a}+e^{-\sigma} m\right)
$$

where $\left\{\bar{\gamma}^{\mu}, \bar{\gamma}^{\nu}\right\}=2 \bar{g}^{\mu \nu}$ and $\bar{g}_{\mu \nu}=g_{\mu \nu} /\left|g_{00}\right|$. Thus, for the square of scalar and spinor operators we have

$$
\bar{H}_{i}^{2}=-\bar{\nabla}^{a} \bar{\nabla}_{a}+e^{-2 \sigma} m^{2}+V_{i} \quad, \quad i=s, d .
$$


The derivatives $\bar{\nabla}_{a}$ are defined in terms of metric $\bar{g}_{a b}$ on the three-dimensional hypersurface $\overline{\mathcal{B}}$. The potential $V_{i}$ is determined by the geometry of the background spaces and by the acceleration $w^{\mu}$ of the Killing observer

$$
\begin{gathered}
V_{s}=\xi \bar{R}+e^{-2 \sigma}(1-6 \xi)\left(\nabla^{\mu} w_{\mu}-w^{\mu} w_{\mu}\right), \\
V_{d}=\frac{1}{4} \bar{R}+e^{-2 \sigma} m \gamma^{\mu} w_{\mu} \\
\bar{R}=e^{-2 \sigma}\left[R+6\left(\nabla^{\mu} w_{\mu}-w^{\mu} w_{\mu}\right)\right] .
\end{gathered}
$$

Note that the operators $\bar{H}_{i}$ can be found if transformation (2.16) of the fields is applied to wave equations (2.2)-(2.3). The new equations obtained in this way can be expressed as equations of the fields on the ultrastatic spacetime with the metric

$$
d \bar{s}^{2}=-d t^{2}+\bar{g}_{a b} d x^{a} d x^{b},
$$

related to the physical metric by the conformal transformation, $\bar{g}_{\mu \nu}=g_{\mu \nu} /\left|g_{00}\right|$. The scalar curvature $\bar{R}$ for this metric is $(2.23)$. Then developing the $(3+1)$-formalism gives single-particle Hamiltonians (2.20). In what follows all quantities calculated with respect to the ultrastatic metric $\bar{g}_{\mu \nu}$ will be denoted with a bar. The single-particle spectra and canonical formulations of the conformally related theories are equivalent. It should be emphasized that in the general case (in the presence of mass $m$ and non-minimal coupling $\xi \neq 1 / 6)$ the theory is not conformal invariant. For scalar fields the conformal invariance occurs only in the case when $m=0$ and $\xi=1 / 6$.

\subsection{Covariant Euclidean formulation}

The canonical formulation of statistical-mechanics is given in accordance with the unitary evolution of the system along the Killing time. It is explicitly related to the " $3+1$ " decomposition and, therefore, it is not explicitly covariant. The covariant Euclidean approach to quantum fields at finite temperatures on stationary backgrounds was suggested by Gibbons and Hawking [58], [59]. This approach proved to be especially useful in application to thermodynamics of black holes [58- 61.

Consider a manifold $\mathcal{M}_{\beta}$ with the Euclidean metric

$$
d s^{2}=g_{\tau \tau} d \tau^{2}+g_{\alpha \beta} d x^{\alpha} d x^{\beta} \quad, \quad 0 \leq \tau \leq \beta
$$

which is obtained from the static Lorentzian metric (2.1) by the Wick rotation $t \rightarrow \tau=i t$, $g_{\tau \tau}=\left|g_{00}\right|$, and imposing the periodicity condition on the imaginary time $\tau$. We assume that the space is asymptotically flat and has the topology $\mathbb{R}^{3}$, so that the topology of $\mathcal{M}_{\beta}$ is $\mathbb{R}^{3} \times S^{1}$.

According to Gibbons and Hawking [58], [59], the partition function $Z^{E}$ and the effective action $W$ for a canonical ensemble of the fields $\Phi$ in an external static gravitational background are defined by the path integral

$$
Z^{E}(\beta)=e^{-W[g, \beta]}=\int[D \Phi] e^{-I[g, \Phi]} .
$$


Here $I[g, \Phi]$ is a classical Euclidean action on the manifold $\mathcal{M}_{\beta}$. Since $g_{\tau \tau}=1$ at spatial infinity, the parameter $\beta$ is the length of $S^{1}$ and hence it has the meaning of the inverse temperature measured at the spatial infinity. Fields $\Phi$ can have either Bose or Fermi statistics. Bose variables are assumed to be periodic in Euclidean time $\tau$ with the period $\beta$, while Fermi fields are antiperiodic. $[D \Phi]$ is a covariant integration measure. For free scalar and Dirac fields the integration in (2.26) gives

$$
\begin{gathered}
W[g, \beta]=W_{s}[g, \beta]+W_{d}[g, \beta] \\
W_{s}[g, \beta]=\frac{1}{2} \log \operatorname{det} \varrho^{-2} L_{s} \quad, \quad W_{d}[g, \beta]=-\log \operatorname{det} \varrho^{-1} L_{d} .
\end{gathered}
$$

The functionals $W_{i}[g, \beta]$ are ultraviolet divergent and it is assumed that their divergencies are regularized. $\varrho$ is an arbitrary renormalization parameter with the dimension of the length, which does not depend on the background metric. In what follows we put $\varrho=1$ for simplicity. If necessary the dependence of the effective actions on $\varrho$ can be easily restored. The operators $L_{i}$ correspond to Eqs. (2.2) and (2.3) and read

$$
L_{s}=-\nabla^{\mu} \nabla_{\mu}+\xi R+m^{2} \quad, \quad L_{d}=\gamma_{5}\left(\gamma^{\mu} \nabla_{\mu}+m\right)
$$

Note that under the Wick rotation from Lorentzian to Euclidean signature the matrix $\gamma_{0}$ has to be replaced by $i \gamma_{0}$. The matrix $\gamma_{5}$ anticommutes with other $\gamma^{\prime}$ s and is normalized as $\gamma_{5}^{2}=1$. Both operators $(2.29)$ are Hermitean with respect to the standard inner product

$$
\left\langle\Phi_{1}, \Phi_{2}\right\rangle=\int d^{4} x \sqrt{g} \Phi_{1}^{+} \Phi_{2}
$$

The Euclidean free energy $F_{i}^{E}[g, \beta]$ is defined by the effective action of the system

$$
F_{i}^{E}[g, \beta]=\beta^{-1} W_{i}[g, \beta]-E_{i}^{0}[g]
$$

Similarly to $F_{i}^{C}[g, \beta]$, the so-defined Euclidean free-energy $F_{i}^{E}[g, \beta]$ vanishes at the zero temperature. This makes the comparison of the two free energies more simple. The vacuum energy

$$
E_{i}^{0}[g]=\lim _{\beta \rightarrow \infty}\left(\beta^{-1} W_{i}[g, \beta]\right)
$$

does not contribute to the entropy. Since the free energy $F_{i}^{E}[g, \beta]$ and the vacuum energy $E_{i}^{0}[g]$ are defined in terms of the covariant Euclidean action $W_{i}[g, \beta]$ this approach is called covariant Euclidean formulation.

\subsection{Relation between canonical and covariant Euclidean formu- lations}

The relation between canonical and Euclidean formulations in the absence of the horizon was discussed by a number of authors [51]-54, [57, 62. 65. 
For the comparison of these two formulations the crucial role is played by the representation of the canonical free energy in terms of the effective action in the ultrastatic space $\overline{\mathcal{M}}_{\beta}$ with the metric

$$
d \bar{s}^{2}=d \tau^{2}+\bar{g}_{\alpha \beta} d x^{\alpha} d x^{\beta} \quad, \quad 0 \leq \tau \leq \beta,
$$

which is conformally related to $\mathcal{M}_{\beta}$. This space is the product $S^{1} \times \overline{\mathcal{B}}$.

Let us define the operators $\bar{L}_{i}$ on $\overline{\mathcal{M}}_{\beta}$ which are conformally related to operators $L_{i}$, Eq. (2.29),

$$
\bar{L}_{s}=e^{-3 \sigma} L_{s} e^{\sigma} \quad, \quad \bar{L}_{d}=e^{-\frac{5}{2} \sigma} L_{d} e^{\frac{3}{2} \sigma} .
$$

It is easy to show that

$$
\begin{gathered}
\bar{L}_{s}=\bar{H}_{s}^{2}-\partial_{\tau}^{2}, \\
\bar{L}_{d}=\gamma_{5} \bar{\gamma}_{\tau}\left(\bar{H}_{d}+\partial_{\tau}\right) \quad, \quad \bar{L}_{d}^{2}=\bar{H}_{d}^{2}-\partial_{\tau}^{2}
\end{gathered}
$$

For these operators one can define the effective actions

$$
\bar{W}_{s}[g, \beta]=\frac{1}{2} \log \operatorname{det} \bar{L}_{s} \quad, \quad \bar{W}_{d}[g, \beta]=-\log \operatorname{det} \bar{L}_{d} \quad .
$$

(For convenience we consider $\bar{W}_{d}$ as the functional of the physical metric $g_{\mu \nu}$.)

For the system with a discrete spectrum one can find with the help of Eqs. (2.35) and (2.36) the following relation between the canonical free energy and the effective action in the ultrastatic space is 57

$$
F_{i}^{C}[g, \beta]=\beta^{-1} \bar{W}_{i}[g, \beta]-\bar{E}_{i}^{0}[g] .
$$

The quantity $\bar{E}_{i}^{0}[g]$ is the vacuum energy for the fields in the ultrastatic space

$$
\bar{E}_{i}^{0}[g]=\eta_{i} \sum_{\omega} d_{i}(\omega) \frac{\omega}{2}
$$

Eqs. (2.38) and (2.39) can be generalized to include the systems with continuous spectra. For this purpose one has to take the limit when the intervals between the levels vanish and to replace sums over $\omega$ with integrals. The derivation of $(2.38)$ and $(2.39)$ can be found in work by Allen [57, see also [66].

It is important to note that $\bar{E}_{i}^{0}[g]$ includes all ultraviolet divergences of the functionals $\bar{W}_{i}[g, \beta]$. Thus the functional $F_{i}^{C}[g, \beta]$ is ultraviolet finite. The geometrical structure of the divergences does not depend on the temperature of the system [54. This is a consequence of a more general property of the quantum field theory whose ultraviolet singularities do not depend on the quantum state of the system [67]. The renormalization of $\bar{W}_{i}[g, \beta]$ is equivalent to renormalization of $\bar{E}_{i}^{0}[g]$.

Equations (2.34) are crucial for finding relation between canonical and Euclidean free energies. The classical actions corresponding to the operators $L_{i}$ and $\bar{L}_{i}$ are

$$
I_{i}^{E}\left[g, \varphi_{i}\right]=\int_{\mathcal{M}_{\beta}} d^{4} x \sqrt{g} \varphi_{i}^{+} L_{i} \varphi_{i} \quad, \quad I_{i}^{C}\left[\bar{g}, \bar{\varphi}_{i}\right]=\int_{\overline{\mathcal{M}}_{\beta}} d^{4} x \sqrt{\bar{g}} \bar{\varphi}_{i}^{+} \bar{L}_{i} \bar{\varphi}_{i} \quad,
$$


where the notation $\varphi_{i}$ is used for scalars $\phi$ or spinors $\psi$. So, as a result of (2.34),

$$
I_{i}^{E}\left[g, \varphi_{i}\right]=I_{i}^{C}\left[\bar{g}, \bar{\varphi}_{i}\right]
$$

for $\bar{\phi}=e^{-\sigma} \phi$ and $\bar{\psi}=e^{-\frac{3}{2} \sigma} \psi$. In case of massless scalars with $\xi=\frac{1}{6}$ or massless spinors fields the operators $L_{i}$ and $\bar{L}_{i}$ have the same form which means that classical theories are conformally invariant. In general case this invariance is broken. However, as it was shown by Dowker and Schofield [64, [65] it is still possible to introduce an auxiliary conformal charge in the classical actions and interpret Eq.(2.41) in terms of a pseudo conformal invariance.

In quantum theory the classical symmetries are known to be broken because of anomalies 67, 68. Thus, the relation between the renormalized actions has the form

$$
W_{i}[g, \beta]=\bar{W}_{i}[g, \beta]+\beta \Omega_{i}[g]
$$

For scalar and spinors fields the anomalous terms $\beta \Omega_{i}[g]$ were found explicitly in [64, [65]. The anomaly is proportional to $\beta$ and so it contributes to the vacuum energy only

$$
E_{i}^{0}[g]=\bar{E}_{i}^{0}[g]+\Omega_{i}[g]
$$

As a result, the free energies $F_{i}^{E}$ and $F_{i}^{C}$, coincide

$$
F_{i}^{E}[g, \beta]=F_{i}^{C}[g, \beta]
$$

Therefore, on the static spacetimes without horizons the covariant Euclidean and canonical formulations of statistical mechanics of quantum fields are equivalent.

The appearance of the anomalous terms in (2.42) can be attributed to non invariance of the integration measure with respect to conformal transformations [69]-[70]. Path integral definition (2.26) of functionals $W_{i}[g, \beta]$ employs the covariant measures $g^{1 / 4} d \phi$ for scalars and $g^{-r_{d} / 2} d \psi^{+} d \psi$ for spinors, where $g=\operatorname{det} g_{\mu \nu}$, and $r_{d}$ is the dimensionality of the spinor representation (see for instance Refs. [71]- [72]). The difference in the prefactors in scalar and spinor measures is related to the different integration rules for Bose and Fermi variables. The integration measure for actions $\bar{W}_{i}[g, \beta]$ is different. It is $\bar{g}^{1 / 4} d \phi$ and $\bar{g}^{-r_{d} / 2} d \psi^{+} d \psi$, for scalars and spinors, respectively, and it is covariant with respect to the ultrastatic background. One can obtain this form of the measure by canonically quantizing the theory [73].

\section{Features related to the horizon}

Many aspects of the computation of the statistical-mechanical entropy of a thermal atmosphere of a black hole are directly related to the presence of the horizon. Consider a general static spacetime with the Killing vector $\zeta=\partial_{t}$ which has a Killing horizon where $\zeta^{2}=0$. At the moment we do not require that the metric obeys the Einstein equations. 
If the surface gravity

$$
\kappa=\left[-\left.\frac{1}{2}\left(\zeta_{\mu ; \nu} \zeta^{\mu ; \nu}\right)\right|_{\zeta^{2}=0}\right]^{1 / 2}
$$

does not vanish, the metric near the horizon can be represented in the form

$$
d s^{2} \simeq-\kappa^{2} \rho^{2} d t^{2}+d \rho^{2}+d \Omega^{2} .
$$

Here $d \Omega^{2}$ is the metric on the two-dimensional bifurcation surface of the horizons where $\zeta^{\mu}=0$. We denote this surface $\Sigma$. In coordinates (3.2) the horizon is located at $\rho=0$. Such form of the metric is general for non-extremal black holes.

In the presence of the horizon the spectrum of quantum fields with respect to the Killing time has a number of important new properties:

1. The single-particle spectrum is continuous even if the system has a finite size, i.e., when there are boundary conditions imposed on the system at the finite distance from the horizon;

2. Regardless of the spin and mass of the fields, the spectrum of $\omega$ runs down to $\omega=0$ and, thus, the usual mass gap is absent;

3. The bifurcation surface $\Sigma$ is invariant under the time-evolution.

The continuity of the spectrum can be easily understood in representation (2.20) for the single-particle Hamiltonians. The operators $\bar{H}_{i}$ are given on the spatial part $\overline{\mathcal{B}}$ of the ultrastatic space (2.24). The space $\overline{\mathcal{B}}$ is always non-compact because the conformal transformation is singular at the event horizon and it moves the points of $\Sigma$ to the spatial infinity in $\overline{\mathcal{B}}$.

The mass gap for the operators $\bar{H}_{i}$ vanishes for the following reason. Equation (2.20) shows that the mass $m_{i}$ of the field does not have any effect near the horizon because of the factor $e^{-2 \sigma}=\left|g_{00}\right|$. The operator $-\bar{\nabla}^{a} \bar{\nabla}_{a}$ has a mass gap which is of the pure geometrical origin. It arises since the space $\overline{\mathcal{B}}$ has asymptotic constant curvature near the horizon. However, on the horizon $V_{s}=-\kappa^{2}, V_{d}=-\frac{3}{2} \kappa^{2}$. These potentials act as a tachionic mass 74, , 75 which exactly cancels the mass gap caused by the curvature of $\overline{\mathcal{B}}$.

Thus, the system we are dealing with behaves near the horizon similar to a massless quantum theory on a non-compact space. It is well known that such theories run into the difficulties related to the infrared divergences. Because of these divergences the densities of eigen-values of quantum-mechanical Hamiltonians $\bar{H}_{i}$ blow up near the horizon. As a result, free energy (2.9) in the canonical formulation diverges at any temperature.

In the presence of the horizon the covariant Euclidean formulation also exhibits new features. In this case the Euclidean section $\mathcal{M}_{\beta}$, Eq. (2.25), of the black hole solution cannot be regular for arbitrary values of $\beta$. Near the surface $\Sigma$ where the Killing vector $\zeta=\partial_{\tau}$ vanishes (the Euclidean horizon) the metric has the form

$$
d s^{2} \simeq \kappa^{2} \rho^{2} d \tau^{2}+d \rho^{2}+d \Omega^{2} \quad, \quad 0 \leq \tau \leq \beta,
$$


which follows from Eq. (3.2). Thus, near this surface $\mathcal{M}_{\beta}$ looks as $\mathcal{C}_{\beta} \times \Sigma$, where $\mathcal{C}_{\beta}$ is a conical space. It is easy to see that the conical singularity disappears and $\mathcal{M}_{\beta}$ is regular space only at the special value $\beta=\beta_{H}=2 \pi \kappa^{-1}$. The corresponding temperature $T_{H}=\beta_{H}^{-1}$ coincides with the Hawking temperature and the corresponding quantum state is known as the Hartle-Hawking vacuum [76]. The physical meaning of $T_{H}$ is that it is the temperature of the Hawking quanta emitted by an evaporating black hole. $T_{H}$ also gives the temperature at which the quantum radiation can be in thermal equilibrium with a black hole.

For $\beta \neq \beta_{H}$ conical singularities result in additional ultraviolet divergences in the effective action on $\mathcal{M}_{\beta}$. Thus, in the presence of the horizon both canonical and covariant Euclidean formulations acquire new divergences. These divergences are of the different origin; they are infrared in the canonical method, and ultraviolet in the covariant Euclidean approach. In Sections 5 and 6 we develop canonical and covariant Euclidean formulation in the presence of the horizon and establish the relation between the divergences in these formulations.

\section{Canonical formulation in the presence of a horizon}

\subsection{Density of levels and its properties}

The divergence of statistical-mechanical quantities in the presence of a horizon is directly related to the infinite growth of the density of states of the Hamiltonians. To investigate this property we follow Ref. [66]. The idea, which is close to the earlier approach of [74], is to relate $d n_{i}(\omega) / d \omega$ to the heat kernel of the operator $\bar{H}_{i}^{2}$. The latter is an elliptic operator and its heat kernel is well known. For a continuous spectrum one has

$$
\operatorname{Tr} e^{-\bar{H}_{i}^{2} t}=\int_{0}^{\infty} d \omega \frac{d n_{i}(\omega)}{d \omega} e^{-\omega^{2} t} .
$$

The density $d n_{i}(\omega) / d \omega$ can be found from (4.1) with the help of the inverse Laplace transform [77. The diagonal matrix elements $\left\langle x\left|\exp \left(-\bar{H}_{i}^{2} t\right)\right| x\right\rangle \equiv\left[\exp \left(-\bar{H}_{i}^{2} t\right)\right]_{\text {diag }}$ are well defined and finite. However, the corresponding trace which involves the integration over the noncompact space $\overline{\mathcal{B}}$ diverges. To understand why it happens it is sufficient to study the behavior of $\left[\exp \left(-\bar{H}_{i}^{2} t\right)\right]_{\text {diag }}$ near the horizon. To estimate the leading asymptotics we can neglect the curvature of the two dimensional surface of the horizon and approximate the black hole metric (3.2) by the metric on the Rindler space

$$
d s^{2}=-\kappa^{2} \rho^{2} d t^{2}+d \rho^{2}+d z_{1}^{2}+d z_{2}^{2} \quad, \quad-\infty<z_{1}, z_{2}<\infty \quad, \quad \rho>0 .
$$

Then the metric on the conformal space $\overline{\mathcal{B}}$

$$
d l^{2}=\kappa^{-2} \rho^{-2}\left(d \rho^{2}+d z_{1}^{2}+d z_{2}^{2}\right)
$$


coincides with the metric of the hyperbolic manifold $\mathbb{H}^{3}$ of constant negative curvature $\bar{R}=-6 \kappa^{2}$. A review of the Laplace operators and their heat kernels on hyperbolic spaces can be found in Refs. [78] and [79]. The eigen-functions of $\bar{H}_{i}^{2}$ are completely determined by the requirement to have the correct decay properties at infinity $\rho \rightarrow \infty$ [75]. Thus, no additional conditions at the horizon $\rho=0$ are needed. When $\rho \rightarrow 0$ the fields become effectively massless and the diagonal elements of the scalar and spinor heat kernels on $\mathbb{H}^{3}$ are known exactly [75], [79]

$$
\left[e^{-t \bar{H}_{s}^{2}}\right]_{\operatorname{diag}}=\frac{1}{(4 \pi t)^{3 / 2}} \quad, \quad\left[e^{-t \bar{H}_{d}^{2}}\right]_{\operatorname{diag}}=\frac{r_{d}}{(4 \pi t)^{3 / 2}}\left(1+\frac{1}{2} \kappa^{2} t\right) .
$$

A summation over the spinor indexes is assumed and it gives the factor $r_{d}$. The geometry essentially differs from the Rindler one far from the horizon and the mass term becomes important. For this reason in general there are corrections to Eqs. (4.4) proportional to the powers of $\rho^{2}$. As it was shown in [66], the structure of these terms can be analysed by using asymptotic properties of the heat kernels on $\overline{\mathcal{B}}$.

Therefore, as follows from Eqs. (4.1) and (4.4), the trace of the operators and their density of states grows as the volume of $\overline{\mathcal{B}}$.

There are several ways how to regularize these divergences. For instance, one can restrict the spatial size of the system. In the given case it means that the region of the physical spacetime where the proper distance to the horizon is smaller than some length, say $\epsilon$, has to be excluded from the consideration. To this aim t'Hooft [18] suggested to impose the Dirichlet boundary conditions on the fields at a surface located outside the horizon and at the proper distance $\epsilon$ from it. t'Hooft's approach is known as the "brick wall" model. A similar but simpler procedure, called the volume cutoff method, was proposed by Frolov and Novikov [31] (see also Refs. [80, [81]). In this method all spatial integrations are cut off at the proper distance $\epsilon$ without imposing a boundary condition.

In the volume cutoff method one effectively cuts a region near the horizon which makes the spacetime incomplete. There exist other types of regularizations which allow one to work on the complete spacetime background. An example is the Pauli-Villars (PV) regularization which was first used for the problem by Demers, Lafrance and Myers [39]. The density $d n / d \omega$ turns out to be finite even in the limit $\epsilon \rightarrow 0$ but it depends on the regulator mass $\mu$ and for $\mu \rightarrow \infty$ it grows as $\mu^{2}$. Another option suggested in [48] is to use the dimensional regularization. The idea of this method is that the power of the leading divergency in Eqs. (4.5) and (4.6) depends on the number of spacetime dimensions. In $D$-dimensional spacetime the leading divergence of the volume of $\overline{\mathcal{B}}$ is $\epsilon^{2-D}$, if $D \neq 2$. One can use $D$ as a regularization parameter for density of states, and take the limit $\epsilon \rightarrow 0$ at $\operatorname{Re} D<2$. The density $d n / d \omega$ then has a pole at $D=4$.

In the volume cutoff method the divergence of $d n / d \omega$ is infrared. In dimensional and PV regularizations the divergence of $d n / d \omega$ can be directly connected with standard ultraviolet divergences. For this reason, we can speak about infrared and ultraviolet limits of the theory depending on which type of regularization is used. 


\subsection{Infrared limit and volume cutoff}

Let us denote the regularized density in the volume cutoff method as $d n_{i}(\omega \mid \epsilon) / d \omega$ and investigate its asymptotics in the limit $\epsilon \rightarrow 0$. After integrating the heat kernels over the region $\rho \geq \epsilon$ and using the inverse Laplace transform in (4.1) one obtains the regularized expression for the divergent part of densities of states [66]

$$
\begin{gathered}
{\left[\frac{d n_{s}(\omega \mid \epsilon)}{d \omega}\right]_{\text {div }}=\frac{1}{4 \pi^{2} \kappa^{3}} \int_{\Sigma}\left\{\omega^{2}\left(\frac{1}{\epsilon^{2}}-\frac{1}{4} \mathcal{P} \ln \frac{\epsilon^{2}}{l^{2}}\right)-\frac{\kappa^{2}}{2} \ln \frac{\epsilon^{2}}{l^{2}}\left[\left(\frac{1}{6}-\xi\right) R-m^{2}\right]\right\},} \\
{\left[\frac{d n_{d}(\omega \mid \epsilon)}{d \omega}\right]_{\text {div }}=r_{d} \frac{1}{4 \pi^{2} \kappa^{3}} \int_{\Sigma}\left\{\omega^{2}\left(\frac{1}{\epsilon^{2}}-\frac{1}{4} \mathcal{P} \ln \frac{\epsilon^{2}}{l^{2}}\right)+\frac{\kappa^{2}}{4 \epsilon^{2}}\right.} \\
\left.-\frac{\kappa^{2}}{2} \ln \frac{\epsilon^{2}}{l^{2}}\left(\frac{1}{8} \mathcal{Q}-\frac{1}{12} R-m^{2}\right)\right\} .
\end{gathered}
$$

The notation $\int_{\Sigma}$ assumes that the integration is performed over the bifurcation surface of the horizons $\Sigma$, so that $\int_{\Sigma} 1=\mathcal{A}$, where $\mathcal{A}$ is the area of $\Sigma$. We imposed an additional cutoff $l$ at large distance $\rho$. The quantity $r_{d}$ is the dimension of the spinor representation, so that $r_{d}=4$ for 4 dimensional Dirac spinors. Let $n_{i}^{\mu}(i=1,2)$ be two unit mutually orthogonal vectors normal to $\Sigma$, then $P^{\mu \nu}=\sum_{i=1}^{2} n_{i}^{\mu} n_{i}^{\nu}$ is a projector onto a two dimensional surface orthogonal to $\Sigma$. The quantities $\mathcal{P}$ and $\mathcal{Q}$ which enter (4.5) and (4.6) are defined as

$$
\mathcal{P}=2 \mathcal{R}-\mathcal{Q} \quad, \quad \mathcal{Q}=P^{\mu \nu} R_{\mu \nu} \quad, \quad \mathcal{R}=P^{\mu \nu} P^{\lambda \rho} R_{\mu \lambda \nu \rho} .
$$

Note that the leading divergence $\epsilon^{-2}$ in Eqs. (4.5)-(4.6) is already present in the Rindler approximation (4.4). The mass and non-zero curvature result in the additional logarithmic divergences $\ln \left(\epsilon^{2} / l^{2}\right)$.

Substituting (4.5) and (4.6) into expression (2.9) for the canonical free energy we obtain

$$
\begin{aligned}
F_{s, \text { div }}^{C}[g, \beta, \epsilon]=- & \frac{1}{\kappa^{3}} \int_{\Sigma}\left\{\frac{\pi^{2}}{180 \beta^{4} \epsilon^{2}}-\left[\frac{\pi^{2}}{720 \beta^{4}} \mathcal{P}+\frac{\kappa^{2}}{48 \beta^{2}}\left(\left(\frac{1}{6}-\xi\right) R-m^{2}\right)\right] \ln \frac{\epsilon^{2}}{l^{2}}\right\} \\
& F_{d, \text { div }}^{C}[g, \beta, \epsilon]=-r_{d} \frac{1}{\kappa^{3}} \int_{\Sigma}\left\{\left(\frac{7 \pi^{2}}{1440 \beta^{4}}+\frac{\kappa^{2}}{192 \beta^{2}}\right) \frac{1}{\epsilon^{2}}\right. \\
& \left.-\left[\frac{7 \pi^{2}}{5760 \beta^{4}} \mathcal{P}+\frac{\kappa^{2}}{96 \beta^{2}}\left(\frac{1}{8} \mathcal{Q}-\frac{1}{12} R-m^{2}\right)\right] \ln \frac{\epsilon^{2}}{l^{2}}\right\}
\end{aligned}
$$

This enables one to calculate other characteristics of canonical ensembles. In particular, one can find the $\epsilon$ divergence of the statistical-mechanical entropy

$$
S_{i, \mathrm{div}}^{C}[g, \beta, \epsilon]=\beta^{2} \frac{\partial F_{i, \mathrm{div}}^{C}[g, \beta, \epsilon]}{\partial \beta}
$$


Evaluated at the Hawking temperature $\beta^{-1}=\beta_{H}^{-1} \equiv \kappa / 2 \pi$ the divergent part of the contributions of bosons and fermions to the entropy is

$$
\begin{gathered}
S_{s, \mathrm{div}}^{C}\left[g, \beta_{H}, \epsilon\right]=\frac{1}{\pi} \int_{\Sigma}\left\{\frac{1}{360 \epsilon^{2}}-\frac{1}{1440}\left[2 \mathcal{R}-\mathcal{Q}+30\left(\frac{1}{6}-\xi\right) R-30 m^{2}\right] \ln \frac{\epsilon^{2}}{l^{2}}\right\} \\
S_{d, \text { div }}^{C}\left[g, \beta_{H}, \epsilon\right]=r_{d} \frac{1}{\pi} \int_{\Sigma}\left\{\frac{11}{1440 \epsilon^{2}}-\frac{1}{5760}\left[7 \mathcal{R}+4 \mathcal{Q}-5 R-60 m^{2}\right] \ln \frac{\epsilon^{2}}{l^{2}}\right\}
\end{gathered}
$$

As we already mentioned, the vacuum energy omitted in (2.6) does not contribute to the entropy. For scalars, the leading divergence $\epsilon^{-2}$ of the entropy is determined by $\beta^{-4}$ term in free energy (4.8). For the spinor fields the situation is different. In order to find $\epsilon^{-2}$ divergence to the entropy one has to know both the leading, $\beta^{-4}$, and subleading, $\beta^{-2}$, terms in free energy (4.9).

To determine the divergences of the free energy and entropy one has to know only the asymptotics of the heat kernel operators [66]. This is not sufficient if one wants to calculate not only the divergencies but the quantities themselves. Important examples, when $d n / d \omega, F^{C}$ and $S^{C}$ can be calculated exactly, were studied in Refs. [74], [75]. The authors considered the case when the spatial part of the spacetime is of the form $\mathcal{B}=\mathbb{R}^{+} \times \Sigma$, where $\Sigma$ is a manifold of constant curvature. In particular, for the Rindler spacetime $\Sigma$ is a two dimensional plane and the density of states of massive scalar fields in Rindler space is 74

$$
\frac{d n_{s}(\omega \mid \epsilon)}{d \omega}=\frac{\mathcal{A}}{4 \pi^{2} \kappa^{3}}\left[\frac{\omega^{2}}{\epsilon^{2}}+\frac{m^{2} \kappa^{2}}{2} \ln \frac{\epsilon^{2} m^{2}}{4}-\frac{m^{2} \kappa^{2}}{2}(1+2 \operatorname{Re} \psi(i \omega / \kappa))\right] .
$$

Here $\psi$ is the logarithmic derivative of the $\Gamma$-function. The terms which vanish as $\epsilon \rightarrow 0$ are omitted. It follows from (4.13) that the role of additional cutoff $l$ in (4.5) and (4.6) is played by $m^{-1}$.

\subsection{Ultraviolet limit}

\section{A. Dimensional regularization}

The dimensional regularization is the simplest scheme which enables one to define $d n / d \omega$ on the complete spacetime. In this regularization one can put $\epsilon=0$ from the very beginning. The quantity $d n / d \omega$ depends on the complex parameter $D$ associated with the dimensionality of the spacetime and it has a pole singularity when $D=4$ [66]

$$
\begin{aligned}
& {\left[\frac{d n_{s}(\omega \mid D)}{d \omega}\right]_{\mathrm{div}}=\frac{\Gamma\left(1-\frac{D}{2}\right)}{(4 \pi)^{D / 2}} \frac{m^{D-4}}{\kappa} \int_{\Sigma}\left[2\left(m^{2}-\left(\frac{1}{6}-\xi\right) R\right)-\frac{\omega^{2}}{\kappa^{2}} \mathcal{P}\right]} \\
& {\left[\frac{d n_{d}(\omega \mid D)}{d \omega}\right]_{\mathrm{div}}=r_{d} \frac{\Gamma\left(1-\frac{D}{2}\right)}{(4 \pi)^{D / 2}} \frac{m^{D-4}}{\kappa} \int_{\Sigma}\left[2\left(m^{2}+\frac{R}{12}-\frac{\mathcal{Q}}{8}\right)-\frac{\omega^{2}}{\kappa^{2}} \mathcal{P}\right]}
\end{aligned}
$$


Table 1:

\begin{tabular}{|c||c||c||c|c|c||c|c|c|}
\hline spin & $d_{1}$ & $f_{1}$ & $q_{1}$ & $q_{2}$ & $q_{3}$ & $p_{1}$ & $p_{2}$ & $p_{3}$ \\
\hline \hline 0 & $\frac{1}{6}-\xi$ & 1 & 1 & -1 & $\frac{5}{2}(1-6 \xi)^{2}$ & $\frac{1}{60}$ & $\frac{1}{6}-\xi$ & 0 \\
\hline \hline$\frac{1}{2}$ & $-\frac{1}{12} r_{d}$ & $-\frac{1}{2} r_{d}$ & $-\frac{7}{8} r_{d}$ & $-r_{d}$ & $\frac{5}{8} r_{d}$ & $-\frac{7}{480} r_{d}$ & $\frac{1}{24} r_{d}$ & $-\frac{1}{16} r_{d}$ \\
\hline
\end{tabular}

These expressions can be used to get the divergences of the canonical free energy for scalars and spinors

$$
\left.F_{\text {div }}^{C}[g, \beta, D]=-\eta \frac{\Gamma\left(1-\frac{D}{2}\right)}{(4 \pi)^{D / 2}} \frac{\pi^{2} m^{D-4}}{3 \kappa \beta^{2}} \int_{\Sigma}\left[f_{1} m^{2}-\left(p_{1} \frac{4 \pi^{2}}{\kappa^{2} \beta^{2}} \mathcal{P}+p_{2} R+p_{3} \mathcal{Q}\right)\right)\right]
$$

The corresponding divergence of the entropy is

$$
\begin{gathered}
S_{i, \mathrm{div}}^{C}[g, \beta, D]=\beta^{2} \frac{\partial F_{i, \mathrm{div}}^{C}[g, \beta, D]}{\partial \beta} \\
\left.S_{i, \mathrm{div}}^{C}[g, \beta, D]=\eta \frac{\Gamma\left(1-\frac{D}{2}\right)}{(4 \pi)^{D / 2}} \frac{2 \pi^{2} m^{D-4}}{3 \kappa \beta} \int_{\Sigma}\left[f_{1} m^{2}-\left(p_{1} \frac{8 \pi^{2}}{\kappa^{2} \beta^{2}} \mathcal{P}+p_{2} R+p_{3} \mathcal{Q}\right)\right)\right] .
\end{gathered}
$$

Constants $f_{1}$ and $p_{k}$ depend on spin and are given in Table 1 . This table contains also other similar constants which we meet later.

\section{B. Pauli-Villars regularization}

It is known that the dimensional regularization reproduces only the logarithmic divergences. For this reason it is also worth studying $F_{\text {div }}^{C}$ in another, more complete regularization. For our purpose it is convenient to use the Pauli-Villars method. In this method for each of the physical field, one introduces 5 additional auxiliary fields: 2 fields with masses $M_{k}$ which have the same statistics as the original field and 3 fields with masses $M_{r}^{\prime}$ which have the wrong statistics. To eliminate the divergences the masses of the auxiliary fields must obey the two restrictions

$$
f(1)=f(2)=0,
$$

where

$$
f(p)=m^{2 p}+\sum_{k} M_{k}^{2 p}-\sum_{r}\left(M_{r}^{\prime}\right)^{2 p}=0 .
$$

\footnotetext{
${ }^{4}$ They are fermions for scalars and bosons for spinors.
} 
These constraints can be resolved by taking $M_{1,2}=\sqrt{3 \mu^{2}+m^{2}}, M_{1,2}^{\prime}=\sqrt{\mu^{2}+m^{2}}, M_{3}^{\prime}=$ $\sqrt{4 \mu^{2}+m^{2}}$ (see [39]). The regularized density of states in this method is

$$
\frac{d n_{i}(\omega \mid \mu)}{d \omega} \equiv \frac{d n_{i}(\omega, m)}{d \omega}+\sum_{k} \frac{d n_{i}\left(\omega, M_{k}\right)}{d \omega}-\sum_{r} \frac{d n_{i}\left(\omega, M_{r}^{\prime}\right)}{d \omega}
$$

The quantities $d n_{i}\left(\omega, M_{k}\right) / d \omega$ and $d n_{i}\left(\omega, M_{r}^{\prime}\right) / d \omega$ are the density of states of the PauliVillars partners. The fields with the wrong statistics give negative contribution in the regularized density. Because the number of such fields equals the number of the fields with the proper statistics the leading $\epsilon$-divergences in relations (4.5) and (4.6) are cancelled. Logarithmic divergences $\ln \epsilon^{2}$ are also canceled because of the constraint $f(p=1)=0$ imposed on masses. As a result, the regularized density of states (4.20) does not contain the divergences when $\epsilon \rightarrow 0$ and it can be defined on the complete background. Since in the presence of regulators $d n_{i}(\omega \mid \mu) / d \omega$ is finite one obtains the same answer as when one uses the dimensional regularization for its calculation. Using Eqs. (4.14) and (4.15) and by taking into account constraints (4.18) one gets

$$
\begin{gathered}
{\left[\frac{d n_{s}(\omega \mid \mu)}{d \omega}\right]_{\mathrm{div}}=\frac{1}{(4 \pi)^{2} \kappa} \int_{\Sigma}\left[2 b+a\left(\frac{\omega^{2}}{\kappa^{2}} \mathcal{P}+2\left(\frac{1}{6}-\xi\right) R\right)\right],} \\
{\left[\frac{d n_{d}(\omega \mid \mu)}{d \omega}\right]_{\mathrm{div}}=r_{d} \frac{1}{(4 \pi)^{2} \kappa} \int_{\Sigma}\left[2 b+a\left(\frac{\omega^{2}}{\kappa^{2}} \mathcal{P}-\frac{R}{6}+\frac{\mathcal{Q}}{4}\right)\right] .}
\end{gathered}
$$

The functions $a$ and $b$ depend on $m$ and $\mu$

$$
\begin{gathered}
a \equiv-\left.\frac{d f}{d p}\right|_{p=0}=-\ln m^{2}-\sum_{k} \ln M_{k}^{2}+\sum_{r} \ln \left(M_{r}^{\prime}\right)^{2}, \\
\left.b \equiv \frac{d f}{d p}\right|_{p=1}=m^{2} \ln m^{2}+\sum_{k} M_{k}^{2} \ln M_{k}^{2}-\sum_{r}\left(M_{r}^{\prime}\right)^{2} \ln \left(M_{r}^{\prime}\right)^{2} .
\end{gathered}
$$

In the Pauli-Villars method $\mu^{2}$ plays the role of the ultraviolet cutoff. In the limit of infinite masses $M_{k}, M_{r}^{\prime}$, i.e. at $\mu \rightarrow \infty, d n_{i}(\omega \mid \mu) / d \omega$ is ultraviolet divergent. In this limit

$$
a \simeq \ln \frac{\mu^{2}}{m^{2}} \quad, \quad b \simeq \mu^{2} \ln \frac{729}{256}-m^{2} \ln \frac{\mu^{2}}{m^{2}} .
$$

Thus, in general $d n / d \omega$ includes both quadratic and logarithmic divergences.

From Eqs. (4.21)-(4.22) one can derive the divergence of the statistical-mechanical free energy

$$
F_{\text {div }}^{C}[g, \beta, \mu]=-\frac{\eta}{48 \kappa \beta^{2}} \int_{\Sigma}\left[b f_{1}+a\left(p_{1} \frac{4 \pi^{2}}{\kappa^{2} \beta^{2}} \mathcal{P}+p_{2} R+p_{3} \mathcal{Q}\right)\right] .
$$

A special case of expression (4.26) for scalar fields on the Reissner-Nordström black hole background was first derived in [39]. The divergent part of the entropy obtained from (4.26) at the Hawking temperature is

$$
S_{\text {div }}^{C}\left[g, \beta_{H}, \mu\right]=\frac{\eta}{48 \pi} \int_{\Sigma}\left[b f_{1}+a\left(2 p_{1} \mathcal{P}+p_{2} R+p_{3} \mathcal{Q}\right)\right] .
$$


By taking into account Eq. (4.25) we can rewrite this expression at large $\mu$ in the form

$$
S_{\mathrm{div}}^{C}\left[g, \beta_{H}, \mu\right]=\frac{\eta}{48 \pi} \int_{\Sigma}\left[c \mu^{2} f_{1}+\left(2 p_{1} \mathcal{P}+p_{2} R+p_{3} \mathcal{Q}-f_{1} m^{2}\right) \ln \frac{\mu^{2}}{m^{2}}\right]
$$

where $c=\ln \frac{729}{256}$. Expression (4.28) has the same structure as the entropy divergences (4.11) and (4.12) in the volume cutoff regularization. It is easy to see that in these regularizations parameters $\epsilon$ and $\mu^{-1}$ correspond to each other. The fact that Pauli-Villars regularization results in a cutoff of the integrals near the horizon at the proper distance comparable to the inverse masses of the fields allows the following interpretation. Near the horizon where the local temperature becomes greater than $\mu$, the massive regulators are thermally excited and because of constraints (4.18) their contribution exactly cancels the contribution of the physical field.

It is interesting that for each field one can find a relation between $\epsilon$ and $\mu^{-1}$ by equating the leading divergencies. After that it is possible to find the connection between $l$ with $m^{-1}$ which makes equal logarithmic divergencies as well. Note, however, that in this identification the relation between $\epsilon$ and $\mu$ is different for fields of the different spins, see Eqs. (4.11), (4.12) and (4.28).

\subsection{WKB computations and the "brick wall" model}

The divergences in statistical-mechanical quantities can be also obtained by using WKB method. This way of computations was suggested by t'Hooft [18] and then used by many authors, see for instance, Refs. [82] 87]. To illustrate this method we consider a scalar field on asymptotically flat spherically symmetric black-hole background

$$
d s^{2}=-g(r) d t^{2}+g^{-1}(r) d r^{2}+r^{2}\left(d \theta^{2}+\sin ^{2} \theta d \varphi^{2}\right)
$$

Here $r \geq r_{h}$, and $r_{h}$ is the horizon radius where $g\left(r_{h}\right)=0$. Schwarzschild and ReissnerNordström black holes are described by metrics of this type. We are interested in modes $\bar{\phi}_{\omega, \ell}(\mathbf{x})$ with energy $\omega$ and angular momentum $\ell$. They are solutions of the eigen-value problem

$$
\bar{H}_{s}^{2} \bar{\phi}_{\omega, \ell}=\omega^{2} \bar{\phi}_{\omega, \ell}
$$

This equation is reduced to one-dimensional problem

$$
\left[-\frac{g^{2}}{r^{2}} \partial_{r}\left(r^{2} \partial_{r}\right)+V_{e f f}^{\ell}(r)-\omega^{2}\right] \bar{\phi}_{\omega, \ell}=0
$$

with the potential

$$
V_{e f f}^{\ell}(r)=-\frac{1}{4}\left(g^{\prime}\right)^{2}+g(r)\left[m^{2}+r^{-2} \ell(\ell+1)\right]
$$

Near the horizon this potential is negative $V_{e f f}^{\ell}\left(r_{h}\right)=-\kappa^{2}$, while at $r \gg r_{h}$ it is positive and $V_{e f f}^{\ell}(r=\infty)=m^{2}$. 
Let us consider now the "brick wall" model [18]. We assume that the "brick wall" Dirichlet condition is imposed at the proper distance $\epsilon$ near the horizon, and $r(\epsilon)$ is the location of the "brick wall" in coordinates (4.29). Equation (4.31) enables one to estimate in the quasiclassical approximation the number of energy levels $n_{s}(\omega \mid \epsilon)$ with the energy smaller than $\omega$

$$
n_{s}(\omega \mid \epsilon)=\frac{1}{\pi} \sum_{\ell=0}^{\ell(\omega)}(2 \ell+1) \int_{r(\epsilon)}^{r_{B}} \frac{d r}{g(r)} \sqrt{\omega^{2}-V_{e f f}^{\ell}(r)} .
$$

Here $\ell(\omega)$ is the maximal angular momentum at which the square root in (4.33) vanishes, $r_{B} \gg r_{h}$ is an additional infrared cutoff.

To estimate the asymptotic behavior of $n_{s}(\omega \mid \epsilon)$ at $\epsilon \rightarrow 0$, we assume that $\omega^{2}$ is large compared to the curvature of the background. Then only the contribution of the large momenta is important and the sum over $\ell$ can be replaced by the integral, which can be easily calculated

$$
n_{s}(\omega \mid \epsilon) \simeq \frac{2}{3 \pi} \int_{r(\epsilon)}^{r_{B}} \frac{r^{2} d r}{g^{2}}\left(\omega^{2}-g m^{2}\right)^{3 / 2} \simeq \frac{\mathcal{A}}{12 \pi^{2} \kappa^{3}}\left(\frac{\omega^{3}}{\epsilon^{2}}+\frac{3}{2} \kappa^{2} m^{2} \omega \ln \frac{\epsilon^{2}}{l^{2}}\right)
$$

In the last equality we put $\mathcal{A}=4 \pi r_{h}^{2}, d \rho=g^{-1 / 2} d r$ and use for the metric (4.29) near the horizon the Rindler approximation (4.2). It is easy to see that Eq.(4.34) gives the same density of energy levels as expression (4.5) for a scalar field with $\mathcal{P}=R=0$.

Thus, at least for the leading divergences there is an agreement between the volume cutoff regularization and the WKB "brick wall" model. This agreement remains if one chooses the Newman boundary condition instead of the Dirichlet one. The numbers $n_{s}(\omega \mid \epsilon)$ for these conditions differ only by a numerical constant [33]. It should be noted that for the physical field the horizon is not a real boundary. From the mathematical point of view it means that the wave functions in Eq.(4.30) with $\omega<m$ can be completely fixed by the requirement to decay fast enough at $r \gg r_{h}$ and no boundary conditions at the cutoff length $\epsilon$ are required. For this reason the volume cutoff method seems to be more appropriate than the "brick wall" approach.

The WKB method can be also used to study the ultraviolet divergences. It was employed, for instance, in [39] in PV regularization. Analogous computations were done in Refs. [83] and [85. Almost all WKB computations are restricted to the case of the scalar field (see, however, Refs. 87 and 88).

\section{Covariant Euclidean formulation}

\subsection{Volume cutoff and high-temperature expansion}

In covariant Euclidean formulation of the theory, the problems related to the divergences on the horizon can be treated in the same way as in the canonical formulation; namely, by 
introducing either the volume cutoff or ultraviolet regularizations. Obviously, comparing the Euclidean and canonical formulations has sense only for the equivalent regularizations.

In the volume cutoff regularization, one effectively cuts a region near the horizon. This makes the spacetime incomplete. As the result, one gets a theory on a static spacetime without the horizon. As we discussed earlier, the covariant Euclidean and canonical formulations are equivalent in this case.

The form of the Euclidean action near the horizon can be found by using the high temperature asymptotics. These asymptotics give a good approximation because the local temperature infinitely grows when approaching the horizon. The high temperature expansion of an effective action was obtained by Dowker and collaborators in [54, [64] and [65] (see also Ref. [89]). The expansion has the form

$$
W_{i}[g, \beta]=\beta \int d^{3} x g^{1 / 2}\left[b_{i}(x, \beta)+h_{i}(x)\right]+W_{i}^{(3)}[g]-\Delta W_{i}
$$

For spinors $W_{d}^{(3)}[g]=0$, while for scalars $W_{s}^{(3)}[g]=-\frac{1}{2} \zeta^{\prime}\left(0 \mid \bar{H}_{i}^{2}\right)$ is determined by the $\zeta$-function of the operator $\bar{H}_{s}^{2}$, see [64], 65]. The quantities $b_{i}$ and $h_{i}$ which enter relation (5.1) are

$$
\begin{gathered}
b_{s}(x, \beta)=-\frac{\pi^{2}}{90 \beta_{l}^{4}}-\frac{1}{24 \beta_{l}^{2}}\left[\left(\frac{1}{6}-\xi\right) R-m^{2}\right]-\frac{a_{s, 2}(x)}{16 \pi^{2}} \ln \frac{\mu \beta_{l}}{2 \pi} \\
b_{d}(x, \beta)=-\frac{7 \pi^{2} r_{d}}{720 \beta_{l}^{4}}+\frac{r_{d}}{48 \beta_{l}^{2}}\left[\frac{1}{12} R+\frac{1}{2}\left(\nabla w-w^{2}\right)+m^{2}\right]+\frac{a_{d, 2}(x)}{16 \pi^{2}} \ln \frac{\mu \beta_{l}}{2 \pi} \\
h_{s}=\frac{1}{2880 \pi^{2}}\left[5 w^{2}\left(w^{2}-2 \nabla w\right)-3(\nabla w)^{2}+R_{\mu \nu} w^{\mu} w^{\nu}-30\left(\left(\xi-\frac{1}{15}\right) R+m^{2}\right) w^{2}\right] \\
h_{d}=\frac{r_{d}}{1440 \pi^{2}}\left[7 w^{2}\left(w^{2}-2 \nabla w\right)-18(\nabla w)^{2}+22 R_{\mu \nu} w^{\mu} w^{\nu}-5\left(R+6 m^{2}\right) w^{2}\right]
\end{gathered}
$$

In these expressions $w^{2}=w^{\mu} w_{\mu}, \nabla w=\nabla^{\mu} w_{\mu}, \beta_{l}(x)=\left|g_{00}\right|^{1 / 2} \beta$ is the inverse local Tolman temperature, $a_{i, 2}(x)$ are the second heat coefficients of the 4-dimensional operators $L_{i}$, Eq.(2.29). The trace over spinor indexes in $a_{d, 2}(x)$ is assumed.

The quantity $\Delta W_{i}$ which enters (5.1) is

$$
\Delta W_{i}=\frac{\beta}{16 \pi^{5 / 2}} \sum_{n=3}^{\infty} c_{i, n} \Gamma\left(n-\frac{3}{2}\right) \zeta_{R}(2 n-3) \bar{a}_{i, n}\left(\frac{\beta}{2 \pi}\right)^{2 n-4} .
$$

Here $c_{s, n}=1, c_{d, n}=1-2^{2 n-3}, \zeta_{R}(z)$ is the Riemann $\zeta$-function 90. $\bar{a}_{i, n}$ are the coefficients of asymptotic expansions of the heat kernels of the operators $\bar{H}_{i}^{2}$. The quantity $\Delta W_{i}$ can also be rewritten in terms of the physical metric and local temperature [64], 65]. Let us emphasize that except the term $W_{i}^{(3)}$ the high temperature expansion (5.1) of the effective action has a local form. The non-local contributions to the finite-temperature effective action was studied in [91]. 
In the volume cutoff method the integration in (5.1) is performed over the spacetime of the black hole till the proper distance $\epsilon$ from the horizon. The action $W_{i}[g, \beta, \epsilon]$ and free energy $F_{i}^{E}[g, \beta, \epsilon]$ diverge when $\epsilon \rightarrow 0$. The divergences are caused only by the terms proportional to $\beta^{-4}$ and $\beta^{-2}$ in the functions $b_{i}(x, \beta)$. The functions $h_{i}$ contribute to the divergence of the vacuum energy. As expected, the divergences in Euclidean and canonical free energies coincide

$$
F_{i, \text { div }}^{C}[g, \beta, \epsilon]=F_{i, \text { div }}^{E}[g, \beta, \epsilon] .
$$

Here the quantities $F_{i \text {,div }}^{C}[g, \beta, \epsilon]$ are determined by Eqs. (4.8), (4.9) and $F_{i, \text { div }}^{E}[g, \beta, \epsilon]$ can be found from the actions (5.1) by subtracting the vacuum energy, see Eq. (2.31).

\subsection{Conical singularities and ultraviolet divergences}

We now consider the definition of the covariant Euclidean effective action on a complete manifold $\mathcal{M}_{\beta}$, i.e., on a manifold with conical singularities . The important feature of this case is that the conical singularities result in additional ultraviolet divergences depending on $\beta$.

It is convenient to introduce the wave operators $\triangle_{i}=-\nabla^{\mu} \nabla_{\mu}+X_{i}$, acting on the scalars and spinors. Here $\nabla$ 's are corresponding covariant derivatives. We have

$$
L_{s}=\triangle_{s}+m^{2} \quad, \quad L_{d}^{2}=\triangle_{d}+m^{2},
$$

where $X_{s}=(1 / 6-\xi) R$ and $X_{d}=\frac{1}{4} R I$. The analysis of Laplacian operators on cones and their heat kernels can be found in Refs. [100]-102]. For physical applications the suitable representation for the Green functions and heat kernels of integer and half-odd-integer spins was given by Dowker [103], 104, see also Ref. [105]. These results are based on the generalization of the representation obtained by Sommerfeld 100 years ago [106].

In general, a one-loop effective action can be defined by the Schwinger-DeWitt representation

$$
W[g, \beta]=\frac{\eta}{2} \log \operatorname{det}\left(\triangle+m^{2}\right)=-\frac{\eta}{2} \int_{\delta^{2}}^{\infty} \frac{d s}{s} e^{-m^{2} s} \operatorname{Tr} e^{-s \triangle},
$$

where $\delta^{2}$ is an ultraviolet cutoff. (For briefness we omit the index $i$ indicating what type of the fields we are considering.) The geometrical structure of the ultraviolet divergences is determined by the first terms in the asymptotic expansion of the heat kernel at small $s$. On manifolds without boundaries it takes the form

$$
\operatorname{Tr} e^{-s \triangle} \approx \frac{1}{(4 \pi s)^{D / 2}}\left(B_{0}+s B_{1}+s^{2} B_{2}+\ldots\right)
$$

where $D$ is the dimension of $\mathcal{M}_{\beta}$. The heat (Hadamard-Minackshisundaram-DeWittSeeley) coefficients $B_{k}$ for $k \geq 1$ can be represented as the sum of two terms

$$
B_{k}=A_{k}+A_{\beta, k}
$$

\footnotetext{
${ }^{5}$ There are several explicit calculations of an effective action on some two-dimensional [92, 80, 93, three-dimensional [94] and four-dimensional [95], 96] spaces with conical singularities. It is worth mentioning that conical singularities appear in a large number of other physical applications including cosmic strings 97], orbifolds in string theory [98] and topological defects in superfluid phases of Helium 3 99].
} 
Here $A_{k}$ has the form of the standard coefficient defined on the regular domain of $\mathcal{M}_{\beta}$. The term $A_{\beta, k}$ is an addition due to conical singularities. This contribution is a functional on $\Sigma$ which depends on the geometrical characteristics of $\mathcal{M}_{\beta}$ near this surface. The first two coefficients $A_{k}$ and $A_{\beta, k}$ have the form

$$
\begin{gathered}
A_{1}=d_{1} \int_{\mathcal{M}_{\beta}-\Sigma} R, \quad A_{\beta, 1}=\frac{\pi}{3 \gamma} f_{1}\left(\gamma^{2}-1\right) \mathcal{A} \\
A_{2}=\frac{1}{180} \int_{\mathcal{M}_{\beta}-\Sigma}\left(q_{1} R^{\mu \nu \lambda \rho} R_{\mu \nu \lambda \rho}+q_{2} R^{\mu \nu} R_{\mu \nu}+q_{3} R^{2}\right) \\
A_{\beta, 2}=\frac{\pi}{3 \gamma} \int_{\Sigma}\left[\left(\gamma^{4}-1\right) p_{1} \mathcal{P}+\left(\gamma^{2}-1\right)\left(p_{2} R+p_{3} \mathcal{Q}\right)\right]
\end{gathered}
$$

where $\gamma=\frac{\beta_{H}}{\beta}$. The coefficients $d_{1}, f_{1}, q_{i}$ and $p_{i}$ for spins $s=0$ and $1 / 2$ can be found in Table 1.

For a scalar field the coefficient $A_{\beta, 1}$ was first found by Cheeger 100, see also Refs. [107, [108]. The coefficients $A_{\beta, 1}$ for spins $1 / 2$ and 1 were obtained in Refs. [109], 110] and for spins $3 / 2$ and 2 in [110]. The coefficient $A_{\beta, 2}$ and general structure of the higher coefficients $A_{\beta, k}$ were analysed in Refs. [11], [112] and [113 for scalars, in Ref. [66], and for spinors in Ref. [114].

The divergent part $W_{\text {div }}[g, \beta]$ of the effective action on $\mathcal{M}_{\beta}$ can be written in different regularizations. It is convenient to begin with the dimensional regularization. For $D \neq 4$ one finds from Eqs. (5.8) and (5.9)

$$
W_{\text {div }}[g, \beta, D]=-\frac{\eta}{2} \int_{0}^{\infty} \frac{d s}{s} e^{-m^{2} s} \frac{1}{(4 \pi s)^{D / 2}}\left(B_{0}+s B_{1}+s^{2} B_{2}\right) .
$$

The Euclidean free energy is obtained from the action by subtracting the vacuum energy (see Eq. (2.31)). On a regular Euclidean manifold the divergences are determined only by the coefficients $A_{0}, A_{1}, A_{2}$ and they are proportional to the period $\beta$. So they do not contribute to the free energy and the entropy. In case of conical singularities the divergences have the form of polynomials in powers of $\beta^{-1}$ because of the additional terms $A_{\beta, k}$. These terms are not removed by subtracting the vacuum part. For this reason the Euclidean free energy is divergent. The divergent part $F_{\text {div }}^{E}[g, \beta, D]$ can be easily found with the help of Eqs. (5.11), (5.13) and (5.14).

As follows from Eq. (4.16), the ultraviolet divergences of the canonical and Euclidean free energies for scalar and spinor fields are identical in the dimensional regularization. This coincidence also takes place in the Pauli-Villars regularization, so one can write

$$
F_{i, \mathrm{div}}^{E}[g, \beta, \delta]=F_{i, \mathrm{div}}^{C}[g, \beta, \delta] \quad, \quad i=s, d \quad .
$$

where $\delta$ is a regularization parameter $(\delta=D-4$ for the dimensional regularization and $\delta=\mu^{-1}$ for PV regularization). As the important consequence of (5.15), the divergences of the entropy in different formulations coincide as well

$$
S_{i, \mathrm{div}}^{E}[g, \beta, \delta]=S_{i, \mathrm{div}}^{C}[g, \beta, \delta] \quad, \quad i=s, d
$$




\subsection{Relation between canonical and Euclidean formulations in the presence of horizons}

Till now we discussed and compared the divergent parts of the free energies calculated in the canonical and covariant Euclidean formulations in the presence of a horizon. In this Section we make remarks concerning the relation between the finite parts of the free energies in these formulations.

As we discussed earlier, the canonical and Euclidean formulations are completely equivalent for static spacetimes without horizons. This conclusion is based on the fact that the effective actions $W[g, \beta]$ and $\bar{W}[g, \beta]$ given on $\mathcal{M}_{\beta}$ and $\overline{\mathcal{M}}_{\beta}$, respectively, are related by a conformal transformation. The Euclidean and canonical free energies obtained from $W[g, \beta]$ and $\bar{W}[g, \beta]$ by subtracting zero temperature parts do not have the divergences. They are free from the anomalies caused by renormalization and as the result $F^{E}$ and $F^{C}$ coincide in the absence of a horizon.

The total functionals $F^{C}[g, \beta, \epsilon]$ and $F^{E}[g, \beta, \epsilon]$ (containing both $\epsilon$-divergent and regular parts) also coincide on the backgrounds with horizons provided one uses the volume cutoff method. In this method the conformal transformation which relates the free energies is well-defined.

The difficulties arise when one compares two formulations on a complete background which includes the horizon. In this case the spaces $\mathcal{M}_{\beta}$ and $\overline{\mathcal{M}}_{\beta}$, have different topologies, $\mathbb{R}^{2} \times \Sigma$ and $S^{1} \times \overline{\mathcal{B}}$, respectively, and the conformal transformation of $\mathcal{M}_{\beta}$ onto $\overline{\mathcal{M}}_{\beta}$ is singular on the bifurcation surface. For this reason the relation between the two formulations requires an additional analysis.

We showed that there exist ultraviolet regularizations which are applicable to both covariant Euclidean and canonical free energies. Let us consider for example a situation when volume cutoff and Pauli-Villars regularizations are applied simultaneously. Then the free energies depend on $\epsilon$ and PV parameter $\mu$. Because the horizon is excluded there is the equality

$$
F_{i}^{E}[g, \beta, \mu, \epsilon]=F_{i}^{C}[g, \beta, \mu, \epsilon] .
$$

As we have shown earlier, the left and right parts of this equality remain finite when $\epsilon$ cutoff is removed. From (5.17) we obtain

$$
F_{i}^{E}[g, \beta, \mu]=\lim _{\epsilon \rightarrow 0} F_{i}^{E}[g, \beta, \mu, \epsilon]=\lim _{\epsilon \rightarrow 0} F_{i}^{C}[g, \beta, \mu, \epsilon]=F_{i}^{C}[g, \beta, \mu]
$$

In the limit $\epsilon \rightarrow 0 F_{i}^{E}$ becomes the functional defined on $\mathcal{M}_{\beta}$, whereas in the same limit $F_{i}^{C}$ is defined on $\overline{\mathcal{M}}_{\beta}$. Equation (5.18) supports the conclusion [83] and 66] that not only the divergencies, but also the complete bare free energies in the covariant Euclidean and canonical formulations are equivalent when one uses the ultraviolet regularization.

There are examples where equality (5.18) can be checked by explicit calculations. For instance, by rewriting the density of levels (4.13) of massive scalar fields in the Rindler space-time in the Pauli-Villars regularization one can find from it the canonical free energy 
and confirm equality (5.18). An explicit derivation of Eq. (5.18) is also possible in two dimensions 83.

Relation (5.18) demonstrates that the partition function $Z^{E}$ defined by Euclidean path integral (2.26) coincides with the canonical partition function $Z^{C}$, Eq. (2.6), including the case of spacetimes with horizons. This fact is very important because it justifies the statistical-mechanical interpretation of the Gibbons-Hawking path integral method [58], [59] when it is applied to black holes.

It should be noted that (5.18) was analysed for scalar and spinor fields only 66]. The relation between the canonical and the covariant Euclidean formulations for high spin fields needs an additional consideration. Some discussion of vector fields can be found in Refs. [115-117].

\subsection{Remarks on rotating and extremal black holes}

So far our discussion was restricted by static black hole geometries. The covariant Euclidean formulation of statistical mechanics for rotating black holes was studied in Refs. [118 and 94. It was shown in 118], an Euclidean manifold which is obtained by the Wick rotation of a stationary geometry with the Killing horizon has a conical singularity similar to the one which appears in static spaces. This singularity results in the one-loop divergence which has the form (4.26). The canonical formulation of the statistical mechanics of quantum fields on a rotating black hole background was discussed in Refs. [119]- [123]. However, the relation between the canonical and covariant Euclidean formulations in this case has not been investigated.

Some remarks are also in order about extremal black holes. The horizon of an extremal black hole has zero surface gravity which indicates that the temperature of such a black hole is zero 124, 125. In the Euclidean theory extremal black holes have the topology of an annulus and there is no conical singularity for an arbitrary period $\beta$ [126], [127]. The statistical mechanics of quantum fields on the background of the extremal black hole has important features which differs it from the non-extremal case. In particular, the leading divergence of the density of levels $\frac{d n}{d \omega}$ of quantum fields is proportional to $e^{L / M}$ where $M$ is the black hole mass and $L$ is the proper distance between the spatial boundary and the location of the cutoff near the horizon [128], [129]. Nevertheless, the ultraviolet type regularizations still can be applied to eliminate the divergencies of the density of states and canonical free energy [39]. Interestingly, the divergences of $\frac{d n}{d \omega}$ and $F^{C}$ in these regularizations have the form which is similar to Eqs. (4.14)-(4.16) and (4.21), (4.22), 4.26). There are no results comparing the canonical and covariant Euclidean formulations of statistical mechanics for extremal black holes.

\footnotetext{
${ }^{6}$ More precisely, to equate $Z^{C}$ and $Z^{E}$ one has to normalize $Z^{E}$ so that to exclude the vacuum energy.
} 


\section{Thermodynamics and statistical mechanics of black holes}

\subsection{Statistical-mechanical entropy and quantum correction to black hole entropy}

Till now we analysed the properties of the statistical-mechanical entropy of quantum fields around a black hole. In the general case this entropy is divergent while the BekensteinHawking entropy is finite. What is the relation between the statistical mechanical entropy of quantum excitations of a black hole and its thermodynamical entropy?

In quantum field theory the quantum corrections are ultraviolet divergent quantities whose divergences are removed by the renormalization of bare coupling constants. As we demonstrated the divergencies of the statistical-mechanical entropy $S_{\text {div }}^{C}$ have ultraviolet

form. It was suggested in 38 that $S_{\text {div }}^{C}$ can be absorbed by the standard renormalition of the Newton constant.

Let us discuss this renormalization approach in more details. The complete information concerning the canonical ensemble of black holes with a given inverse temperature $\beta$ at the boundary is contained in the partition function $Z(\beta)$ given by the Euclidean path integral 59

$$
Z(\beta)=\int[D \Phi] \exp (-I[\Phi])
$$

Here the integration is taken over all fields including the gravitational one that are given on the Euclidean section and are periodic (or antiperiodic) in the imaginary time coordinate $\tau$ with period $\beta$. The quantity $\Phi$ is understood as the collective variable describing the fields. In particular, it contains the gravitational field. Here $[D \Phi]$ is the measure of the space of fields $\Phi$ and $I$ is the Euclidean action of the field configuration. The action $I$ includes the Euclidean Einstein action. The state of the system is determined by the choice of the boundary conditions on the metrics that one integrates over. For the canonical ensemble of the gravitational field inside a spherical box of radius $r_{0}$ at temperature $T$ one must integrate over all the metrics inside $r_{0}$ which are periodically identified in the imaginary time direction with period $\beta=T^{-1}$. Such a partition function must describe in particular the thermal ensemble of black holes. The partition function $Z$ is related to the effective action $\Gamma=-\ln Z$. The free energy $F$ is defined as $F=\beta^{-1} \Gamma=-\beta^{-1} \ln Z$.

By using the stationary-phase approximation one gets

$$
\beta F \equiv \Gamma=I\left[\Phi_{0}\right]+W+\ldots
$$

Here $\Phi_{0}$ is the (generally speaking, complex) solution of classical field equations for action $I[\Phi]$ obeying the required periodicity and boundary conditions. Besides the tree-level contribution $I\left[\Phi_{0}\right]$, expression (6.2) includes also one-loop corrections $W$, connected with the contributions of the fields perturbations on the background $\Phi_{0}$, and higher order 
terms in loop expansion, denoted by (...). For free fields, $W$ is a one-loop effective action computed in the covariant Euclidean formulation of the theory, see Eqs. (2.28).

The one-loop divergences appearing in $W$ can be absorbed by the renormalization of the couplings of the initial classical action $I$. To this aim the latter is chosen in the form

$$
\begin{gathered}
I\left(G_{B}, \Lambda_{B}, c_{B}^{i}\right)=\int d^{4} x \sqrt{g} L \\
L=\left[-\frac{\Lambda_{B}}{8 \pi G_{B}}-\frac{R}{16 \pi G_{B}}+c_{B}^{1} R^{2}+c_{B}^{2} R_{\mu \nu} R^{\mu \nu}+c_{B}^{3} R_{\alpha \beta \mu \nu} R^{\alpha \beta \mu \nu}\right] .
\end{gathered}
$$

In the presence of one-loop divergences the stationary-phase approximation procedure is modified as follows. Denote by $W_{\text {div }}$ the UV-divergent part of the one-loop effective action $W$. Then the renormalized quantities are defined as

$$
I_{\mathrm{ren}} \equiv I\left(G_{\mathrm{ren}}, \Lambda_{\mathrm{ren}}, c_{\mathrm{ren}}^{i}\right)=I\left(G_{B}, \Lambda_{B}, c_{B}^{i}\right)+W_{\mathrm{div}} \quad, \quad W_{\mathrm{ren}}=W-W_{\mathrm{div}} \quad .
$$

Now the starting point of the semiclassical approximation is in finding the extremal of the renormalized action $I_{\text {ren }}$. Since for this background $W_{\text {ren }}$ is finite and proportional to $\hbar$, this part of the action describes small quantum corrections.

The key observation of the renormalization approach is that $W_{\text {div }}$ has the same structure as (6.3)-(6.4) and hence $W_{\text {div }}$ can be absorbed by simple redefinition of the coupling constants in $I\left(G_{B}, \Lambda_{B}, c_{B}^{i}\right)$. In other words, $I_{\text {ren }}$ is identical to the initial classical action $I$ with the only change that the bare coefficients $\Lambda_{B}, G_{B}$, and $c_{B}^{i}$ are substituted by their renormalized versions $\Lambda_{\text {ren }}, G_{\text {ren }}$, and $c_{\text {ren }}^{i}$. $W_{\text {div }}$ can be found by using relation (5.9) and expressions (5.10)-(5.12) for the heat kernel coefficients of the corresponding Laplace operators (on regular backgrounds). The relation between bare and renormalized couplings depends on the regularization. For instance, in PV regularization the renormalization of the Newton constant for the non-minimally coupled scalar field results is

$$
\frac{1}{G_{\mathrm{ren}}}=\frac{1}{G_{B}}+\frac{c}{2 \pi}\left(\frac{1}{6}-\xi\right) \mu^{2}
$$

where $c=\ln \frac{729}{256}$ and $\mu$ is the PV cutoff.

The free energy $F(\beta)=\beta^{-1} \Gamma$ being expressed in terms of the renormalized constants is finite. By calculating $F(\beta)$ on the black hole instanton one finds the one-loop free energy of a black hole. The "observable" thermodynamic entropy of a black hole $S^{T D}$ [37] has the standard form $S^{T D}=\beta^{2} d F(\beta) / d \beta$. If we neglect for a while by the logarithmic divergencies and put $\Lambda_{\text {ren }}=0$ we obtain

$$
S^{T D}=S^{B H}\left(G_{\text {ren }}\right)+O(\hbar)
$$

Here $S^{B H}\left(G_{\mathrm{ren}}\right)$ is the Bekenstein-Hawking entropy in the theory of general relativity with the Newton constant $G_{\text {ren }}$. The terms $O(\hbar)$ represent finite quantum corrections proportional to $\hbar$. Equations (6.6) and (6.7) can be compared with the statistical mechanical 
entropy $S^{C}$ of quantum fields. For a scalar field the leading divergency of $S^{C}$ can be found from (4.28)

$$
S_{\mathrm{div}}^{C}=\frac{c}{48 \pi} \mu^{2} \mathcal{A}
$$

Thus, by using Eq. (6.6) we find

$$
S^{B H}\left(G_{\mathrm{ren}}\right)=S^{B H}\left(G_{\mathrm{B}}\right)+S_{\mathrm{div}}^{C}-Q_{\mathrm{div}},
$$

where the quantity $Q_{\text {div }}=\xi c \mu^{2} \mathcal{A} /(2 \pi)$ appears when a scalar field is non-minimal coupled with the curvature. In the next Section we show that Eq. (6.9) preserves its form when not only the leading but all the divergencies are included.

Equation (6.7) explicitly demonstrates that the "observable" Bekenstein-Hawking entropy contains the statistical-mechanical entropy of black-hole's quantum excitations as its part, but in the general case it does not coincides with it. For non-minimal coupling an additional term $Q$ is present. Even in the absence on nonminimal coupling when $Q=0$, the presence of the bare pure geometrical contribution $S^{B H}\left(G_{\mathrm{B}}\right)$ evidently excludes the possibility to identify $S^{B H}\left(G_{\text {ren }}\right)$ with $S_{\text {div }}^{C}$ which has clear statistical mechanical meaning. Moreover, in order to have finite value of $S^{B H}\left(G_{\text {ren }}\right)$ one needs to assume that pure geometrical "entropy" $S^{B H}\left(G_{\mathrm{B}}\right)$ is infinite and negative. For this reason the idea to relate $S^{B H}$ with quantum excitations does not work, at least in the standard renormalization approach. The way out of this problem is to restrict oneself by considering special class of the theories where $S^{B H}\left(G_{\mathrm{B}}\right)=0$. It happens when $G_{\mathrm{B}}^{-1}=0$, and hence initially gravity is not dynamical. The dynamics of the gravitational field arises as the result of quantum effects. The induced gravity is an example of such a theory.

\section{General renormalization and the Noether charge}

Before discussing the models of induced gravity we make comments on the generalization of Eq. (6.9). Let us consider the case when terms quadratic in curvature are preserved in the renormalized action. In this case the classical black hole entropy is (see Refs. $130-133$ )

$$
S^{B H}\left(G_{\mathrm{ren}}, c_{\mathrm{ren}}^{i}\right)=\frac{1}{4 G_{\mathrm{ren}}} \mathcal{A}-\int_{\Sigma} \sqrt{\sigma} d^{2} \theta\left(8 \pi c_{\mathrm{ren}}^{1} R+4 \pi c_{\mathrm{ren}}^{2} \mathcal{Q}+8 \pi c_{\mathrm{ren}}^{3} \mathcal{R}\right) .
$$

The integral in (7.1) is taken over the bifurcation surface of the horizon. The first term in the r.h.s. of Eq. (7.1) is the Bekenstein-Hawking entropy, and other terms are additions because of the high curvature terms in the action.

For PV and dimensional regularizations in the model with the scalar and spinor fields the relation (6.9) takes the form]

$$
S^{B H}\left(G_{\mathrm{ren}}, c_{\mathrm{ren}}^{i}\right)=S^{B H}\left(G_{\mathrm{B}}, c_{\mathrm{B}}^{i}\right)+S_{\mathrm{div}}^{C}-Q_{\mathrm{div}} .
$$

\footnotetext{
${ }^{7}$ In Section 4.3 we pointed out that the divergencies $S_{\text {div }}^{C}$ of the statistical-mechanical entropy in the
} 
In PV regularization $S_{\text {div }}^{C}$ is determined by expression (4.28). The quantity $Q_{\text {div }}$ appears for nonminimally coupled scalars,

$$
Q_{\mathrm{div}}=\xi \frac{1}{8 \pi} \int_{\Sigma}\left[b+a\left(\frac{1}{6}-\xi\right) R\right] .
$$

The coefficients $a, b$ depend on PV cutoff $\mu$ and are given by Eqs. (4.23), (4.24). When $Q_{\text {div }}=0$ the general proof of (7.2) was given in Refs. [39] and [40] (see also Refs. [42], 138, 139 and 109).

For scalar fields with the nonminimal coupling, quantity (7.3) can be written as

$$
Q_{\mathrm{div}}=2 \pi \xi \int_{\Sigma}\left\langle\hat{\phi}^{2}\right\rangle_{\mathrm{div}}
$$

where it is assumed that the fluctuation of the scalar field $\left\langle\hat{\phi}^{2}\right\rangle$ is computed in PV regularization. Relation (7.4) was first found by Solodukhin [41] with the help of the Euclidean formulation of the theory with conical singularities.

The reason why quantity (7.4) appears in formula (7.2) is the following. In the presence of scalar fields with nonminimal couplings the Bekenstein-Hawking entropy includes the additional term $Q=2 \pi \xi \int_{\Sigma} \phi^{2}$, where $\phi$ is the classical field, see [133]. In quantum theory the quantity $Q$ becomes the operator whose average has the divergent part which coincides with $Q_{\text {div }}$. In the one-loop approximation $Q_{\text {div }}$ determines the quantum correction to $S^{B H}$ because of the non-minimal coupling.

It was shown recently by Wald et al. [131]-133, [140], 141] that the classical black hole entropy in diffeomorfism invariant theories of gravity can be interpreted as the Noether charge. In Appendix we demonstrate that $Q$ coincides with the Noether charge for the nonminimally coupled matter fields propagating on the fixed curved background and construct the corresponding Noether current. We also show that

$$
Q=\frac{2 \pi}{\kappa}(H-E),
$$

where $\kappa$ is the surface gravity, $H$ is the canonical Hamiltonian of the fields and $E$ is the energy of the fields obtained from the stress-energy tenor, see definitions (A.2) and (A.5) of Appendix. The latter relation plays an important role in the models of induced gravity.

volume cutoff method also take the ultraviolet form if one identifies the volume cutoff parameter $\epsilon$ with an ultraviolet cutoff. The problem is that each field species requires its particular relation between $\epsilon$ and $\mu$, and there is no universal relation which enables one to remove the divergencies from all fields. For instance, for scalars the relation between the volume cutoff and PV parameters looks as $\epsilon^{-2}=c \frac{15}{2} \mu^{2}$, see Eqs. (4.11), 4.28), while for spinors the same relation has to be $\epsilon^{-2}=c \frac{15}{11} \mu^{2}$. This indicates that the volume cutoff parameter is not a truly covariant ultraviolet regulator. Thus, regarding the renormalization problem, the volume cutoff and "brick-wall" methods run into the difficulty. Some other difficulties of these methods are discussed in Refs. [134-137. For two-dimensional black hole models the relation between the "brick wall" results and the renormalized quantum correction to black hole entropy is discussed in Refs. 80 and 93 . 


\section{Black hole entropy in induced gravity}

The theory of induced gravity was suggested by Sakharov 46] long ago. The low-energy gravitational effective action $\Gamma[g]$ in this theory is defined as a quantum average of the constituent fields $\Phi$ propagating in a given external gravitational background $g$

$$
\exp (-\Gamma[g])=\int[D \Phi] \exp (-I[g, \Phi])
$$

The Sakharov's basic assumption is that the gravity becomes dynamical only as the result of quantum effects of the constituent fields. As a result, we have theories of the special type, namely theories with $I[g]=0$. The gravitons in such a picture are analogous to the phonon field describing collective excitations of a crystal lattice in the low-temperature limit of the theory. In the general case, each particular constituent field in (8.1) gives a divergent contribution to the effective action $\Gamma[g]$. In the one loop approximation the divergent terms are local and of the zero order, linear and quadratic in curvature. In the induced gravity the constituents obey additional constraints, so that the divergences cancel each other. It is also assumed that some of the fields have masses comparable to the Planck mass and the constraints are chosen so that the induced cosmological constant vanishes. As the result the effective action $\Gamma[g]$ is finite and in the low-energy limit it has the form of the Einstein-Hilbert action

$$
\Gamma[g]=-\frac{1}{16 \pi G}\left(\int_{\mathcal{M}} d V R+2 \int_{\partial \mathcal{M}} d v K\right)+\ldots
$$

where Newton's constant $G$ is determined by the masses of the heavy constituents 8 . The dots in (8.2) indicate possible higher curvature corrections to $W[g]$ which are suppressed by the power factors of $m_{i}^{-2}$ when the curvature is small. The vacuum Einstein equations $\delta W / \delta g^{\mu \nu}=0$ are equivalent to the requirement that the vacuum expectation values of the total stress-energy of the constituents vanishes

$$
\left\langle\hat{T}_{\mu \nu}\right\rangle=0
$$

The value of the Einstein-Hilbert action (8.2) calculated on the Gibbons-Hawking instanton determines the classical free energy of the black hole, and hence gives the BekensteinHawking entropy $S^{B H}$.

Consider a model of induced gravity 48 that consists of a number of scalar fields with masses $m_{s}$ and a number of Dirac fermions with masses $m_{d}$. Scalar fields can have non-minimal couplings $\xi_{s}$. It is convenient to introduce the following two functions

$$
p(z)=\sum_{s} m_{s}^{2 z}-4 \sum_{d} m_{d}^{2 z}, \quad q(z)=\sum_{s} m_{s}^{2 z}\left(1-6 \xi_{s}\right)+2 \sum_{d} m_{d}^{2 z}
$$

\footnotetext{
${ }^{8}$ As an another realization of the idea of induced gravity it is worth mentioning the approach by Adler, for a review see Refs. 142, 143. According to Adler, the induced Newton constant may arise as a result of the dimensional transmutation because of the dynamical symmetry breaking in the underlying scale invariant theory. This picture is different from the mechanism under discussion.
} 
Direct calculations show that the induced cosmological constant vanishes when

$$
p(0)=p(1)=p(2)=p^{\prime}(2)=0 .
$$

The induced gravitational coupling constant $G$ is finite if the following constraints are satisfied

$$
q(0)=q(1)=0 .
$$

Relations (8.5) are satisfied for theories with supersymmetric massive multiplets for which $p(z)=0$. Equations (8.6) form a linear system defining $\xi_{s}$.

The presence of the non-minimally coupled constituents is important. In this case it is possible to satisfy constraints (8.6) on the parameters $m_{s}, m_{d}$ and $\xi_{s}$ which guarantee the cancellation of the leading ultraviolet divergencies of the induced gravitational action $\Gamma[g]$. The induced Newton's constant in this model is [48]

$$
\frac{1}{G}=\frac{1}{12 \pi}\left(\sum_{s}\left(1-6 \xi_{s}\right) m_{s}^{2} \ln m_{s}^{2}+2 \sum_{d} m_{d}^{2} \ln m_{d}^{2}\right) .
$$

Let us analyze now the entropy of a Schwarzschild black hole in the induced gravity. If constraints (8.5) and (8.6) are satisfied the induced action has logarithmically divergent terms only. However these terms are quadratic in curvature and on the Schwarzschild background they can be neglected because they do not depend on the geometry. Consider the difference $S^{C}-Q$, where $S^{C}$ is the statistical-mechanical entropy of the scalar and spinor massive fields (constituents) and $Q$ is the Noether charge which appears because of non-minimal couplings of the scalar constituents. $S^{C}$ and $Q$ are assumed to be regularized according to the same prescription, say, by PV method. In the low-energy limit of the theory one can calculate these quantities in the leading order in $m_{i}^{2}$. In this approximation $S^{C}$ coincides with the part $S_{\text {div }}^{C}$ determined in PV-regularization by Eq. (4.27). Analogously, the Noether charge is approximated by $Q_{\text {div }}$, which can be found from Eq. (7.3). It is easy to check that for a Schwarzschild black hole the leading divergencies in $S^{C}-Q$ are cancelled and from Eqs. (4.27) and (7.3) one obtains

$$
S^{C}-Q \simeq S_{\text {div }}^{C}-Q_{\text {div }}=\frac{1}{4 G} \mathcal{A}+C .
$$

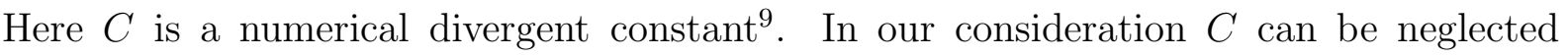
because it does not depend on the black hole geometry. Thus, up to this numerical addition, $S^{C}-Q$ agrees precisely with the induced Bekenstein-Hawking entropy $S^{B H}=$ $\frac{1}{4 G} \mathcal{A}$. The result (8.8) can be also obtained by using the dimensional regularization苗.

\footnotetext{
${ }^{9}$ The divergence of $C$ is a specific property of the given simplified model which is not free from some logarithmic divergencies. In a more complicated model including, for instance, vector massive fields $C$ can be made finite.

${ }^{10}$ It should be noted, however, that the check of Eq. (8.8) in the volume cutoff regularization may meet a difficulty [144]. Presumably, it happens because this regularization makes the background space incomplete, and so using expression (7.4) for $Q$ in the entropy becomes unjustified.
} 
As follows from (8.8), the Bekenstein-Hawking entropy is determined by the leading order of the statistical-mechanical entropy of the heavy constituent fields. The quantity $S_{\text {div }}^{C}$ comes out as the result of integration over a narrow layer located near the horizon and having the size of the order of the Comtpton wave length of the constituents, which is comparable to the Planck length. Thus, the black hole entropy depends only on the local properties of the horizon. This conclusion implies that relation (8.8) must be also valid for a generic static or stationary black holes.

Another conclusion which can be drawn from (8.8) is that the black hole entropy $S^{B H}$ is not identical to the statistical-mechanical entropy $S^{C}$. Actually, $S^{B H}$ is a finite quantity, while $S^{C}$ diverges because each of the constituents gives a positive divergent contribution into it. In Eq. (8.8) the divergences of $S^{C}$ are eliminated by subtraction of the charge $Q$. As it can be shown, in the model in question $Q$ has positive divergencies. Thus, the presence of the fields with non-minimal couplings which is imperative in order to make the theory ultraviolet finite also provides the subtraction of the divergencies of $S^{C}$.

Is there a statistical-mechanical explanation why the Noether charge $Q$ enters relation (8.8)? A possible mechanism was suggested in Ref. [49]. The induced gravity explains the black hole entropy by relating it to statistical-mechanical entropy of the constituents. As the result of the backreaction effect, the black hole geometry responds to quantum fluctuations of these fields. One consequence of this effect is that fluctuations of the energy of fields $E$ near the average value $E=0$ cause the fluctuations of the black hole mass near its average value $M$. The black hole entropy $S^{B H}$ determines the number density of the states in the interval $(M, M+\Delta M)$ so the quantity $\exp S^{B H}$ coincides with the degeneracy $\nu(M)$ of the black hole mass spectrum. Now it is easy to show by using differential mass formula [ []] (see also Eq. (11.2.48) of Ref. [145]) that in the leading order in the Planck constant the change $\Delta M$ of the black hole mass coincides with the change $\Delta E$ of the energy of the constituents. Therefore, the problem of finding the degeneracy of the black hole mass spectrum can be reduced to the problem of finding the spectrum of the energy of the constituent fields, which is more simple. In particular, the black hole entropy can be related with the number density $\nu(E)$ of $E$ as $e^{S^{B H}}=\nu(E=0)$.

Contrary to $S^{B H}$, the statistical-mechanical entropy $S^{C}$ is related to the spectrum of the Hamiltonian $H$ generating translations of the system along the Killing time. $H$ differs from $E$ by the Noether charge $Q$. That is why $S^{B H}$ and $S^{C}$ are different and related by Eq. (8.8) 49.

Why the density number of states with the energy $E$ is finite, while the number of states of $H$ diverges even in the ultraviolet finite theory? The answer to this question is related to the specific property of the quantum system in the presence of the Killing horizon. As we pointed out, the spectrum of frequencies of the single-particle excitations in this case does not have the mass gap. In other words, there exist modes with negligibly 
small frequencies, so called soft modes [49. An arbitrary number of the soft modes can be added to such a system without changing its canonical energy $H$. It is the soft modes which result in additional infinite degeneracy of the spectrum of the Hamiltonian $H$. However, contribution of the soft modes to the energy $E$ is not zero and the spectrum of $E$ doesn't have the redundant degeneracy.

The reason why the soft modes contribute to $E$ is that $E$ differs from $H$ by the Noether charge $Q$. In fact, $Q$ is determined by the soft modes only. To see this, it is sufficient to approximate the black hole geometry (4.2) near the horizon by the Rindler space (4.2). According to Eq. (7.4), $Q$ is determined by the average of the scalar correlator $\left\langle\hat{\phi}^{2}\right\rangle$ on the bifurcation surface $\Sigma$. In the Rindler approximation the correlator can be easily computed 49

$$
\begin{gathered}
\left\langle\hat{\phi}(x) \hat{\phi}\left(x^{\prime}\right)\right\rangle=\operatorname{Tr}\left[\hat{\rho} \hat{\phi}(x) \hat{\phi}\left(x^{\prime}\right)\right] \\
=\int_{0}^{\infty} d \omega \int d^{2} k\left[n_{\omega} \phi_{\omega, k}^{*}(x) \phi_{\omega, k}\left(x^{\prime}\right)+\left(n_{\omega}+1\right) \phi_{\omega, k}(x) \phi_{\omega, k}^{*}\left(x^{\prime}\right)\right] .
\end{gathered}
$$

Here $n_{\omega}=(\exp (2 \pi \omega / \kappa)-1)^{-1}$ is the number of particles at the Hawking temperature with the energy $\omega$. The functions $\phi_{\omega, k}(x)$ are the eigen functions of the single-particle Hamiltonian $H_{s}$ determined by Eq. (2.11). On the Rindler space (4.2)

$$
\phi_{\omega, k}(x)=\frac{1}{4 \pi^{3} \kappa^{1 / 2}}(\sinh (\pi \omega / \kappa))^{1 / 2} K_{i \omega / \kappa}\left(\rho\left(m^{2}+k_{j}^{2}\right)^{1 / 2}\right) e^{-i \omega t} e^{-i k_{j} z^{j}}
$$

where $k^{i}$ and $z^{i}$ are the momentum and the coordinate along the horizon. $K_{i \omega / \kappa}$ are the modified Bessel functions. When the proper distance to the horizon $\rho$ goes to zero $K_{i \omega / \kappa}\left(\rho\left(m^{2}+k_{j}^{2}\right)^{1 / 2}\right)$ behave as the delta function $\delta(\omega)$. Thus, when one of the arguments of correlator (8.9) is placed on the horizon only the contribution of the soft modes survives. By using PV regularization and taking the limit $\rho \rightarrow 0$ in (8.9) one gets $Q_{\text {div }}$ in form (7.3) with $R=0$.

The induced gravity proposes the following statistical-mechanical interpretation of black hole entropy formula (8.8). $S^{B H}$ is determined by the density number of physical states corresponding to the given black hole mass $M$. The physical states are related to the states of the excitations of the constituents fields in the black hole exterior which result in the fluctuation of the black hole mass. The space of physical states is obtained by factorizing the space of all thermal excitations over the subspace of the soft modes. This removes the additional degeneracy and makes the number density of the physical states finite. The factorization over the soft modes is equivalent to subtracting of the Noether charge from the entropy $S^{C}$, which reduces the latter to $S^{B H}$. As was pointed out in [49], there is a similarity between this mechanism and gauge theories, soft modes playing the role of the pure gauge degrees of freedom. 


\section{Summary}

This review is devoted to the description of thermal ensembles of quantum fields in a spacetime of a black hole. This study was stimulated by the attempts to give the statisticalmechanical explanation of the Bekenstein-Hawking the entropy. This is the key problem of black hole physics.

The main difficulty of the statistical mechanics of quantum fields near black holes is connected with additional thermal (infrared) divergences. In the presence of these divergences the relation between canonical and covariant Euclidean formulations of the theory requires reconsideration. In this review we payed a special attention to the analysis of the divergences and methods of their regularizations. It was demonstrated that the canonical and covariant Euclidean formulations are equivalent, and in the same regularization the divergences in the both formulations are identical. An important property of the problem is that the thermal divergences in one regularization take the form of ultraviolet divergences in the other regularization. This duality is crucial for the discussion of the black hole entropy. Thermal divergences which contribute to the black hole entropy are connected with ultraviolet one-loop divergences which renormalize gravitational coupling constants.

We analyzed the problem of black hole entropy and demonstrated it cannot be solved in a theory of gravity within the standard scheme of renormalizations. The renormalization requires initial bare entropy, which is of pure geometrical origin and (in the absence of non-minimal coupling) is infinite negative quantity. However, if the bare classical (tree level) gravity is absent the Bekenstein-Hawking entropy $S^{B H}$ can be directly related to the statistical-mechanical entropy $S^{C}$ of quantum black hole excitations. New important feature is that this relation necessarily contains the Noether charge $Q$ for non-minimally coupled fields. In one-loop ultraviolet-finite theories without bare gravity there exist the relation between $S^{B H}$ and $S^{C}$ :

$$
S^{B H}=S^{C}-Q
$$

These theories belong to the class of so-called induced gravity theories. In these theories gravity is induced as the result of collective quantum excitations of heavy constituents of the Planckian mass. The same constituent fields which generate low energy gravity are responsible for the entropy $S^{B H}$ of a black hole.

The analysis of concrete models shows that the relation (9.1) is the result of the consistency of the theory. The Noether charge $Q$ determines the difference between the energy $E$ of the system and the value of the Hamiltonian $H$. The entropy $S^{B H}$ describes the degeneracy of states of the black hole with respect to its mass. It can be related to the degeneracy of the system of constituents with respect to its energy $E$. The latter differs from the degeneracy of the Hamiltonian $H$. Formula (9.1) takes into account this fact.

In this approach fields (constituents) which contribute into the entropy are to be considered as fundamental. One can expect that such fields arise in the fundamental 
theory of quantum gravity, for instance, in the string theory. This mechanism is not known at present, but its possible existence might explain universality of the entropy of black holes.

Acknowledgements: This work was partially supported by the Natural Sciences and Engineering Research Council of Canada. One of the authors (V.F.) is grateful to the Killam Trust for its financial support. 


\section{A Energy, Hamiltonian and Noether charge}

To explain the meaning of the Noether charge $Q$ discussed in Section 7 let us consider a classical real scalar field $\phi$ on the static background with the action

$$
I[\phi, g]=-\frac{1}{2} \int\left(\phi^{, \mu} \phi_{, \mu}+m^{2} \phi^{2}+\xi R \phi^{2}\right) \sqrt{-g} d^{4} x \quad .
$$

The energy $E$ of the field in the $3 \mathrm{D}$ region $\mathcal{B}$ is determined by the stress-energy tensor $T_{\mu \nu}$

$$
E=\int_{\mathcal{B}} T_{\mu \nu} \zeta^{\mu} d \sigma^{\nu}
$$

Here $d \sigma^{\nu}$ is the future directed vector of the volume element on $\mathcal{B}$ and $\zeta^{\mu}$ is the time-like Killing vector. The stress-energy tensor is obtained by the variation of action (A.1)

$$
T_{\mu \nu}=\frac{2}{\sqrt{-g}} \frac{\delta I[g]}{\delta g^{\mu \nu}} .
$$

In a static spacetime the energy $E$ is conserved on the equations of motion of the field $\phi$ (i.e., $E$ does not depend on the choice of $\mathcal{B}$ ). In addition to the stress-energy tensor (A.3), one can define the canonical stress-energy tensor

$$
\left(T^{C}\right)_{\mu \nu}=\phi_{, \mu} \frac{\partial \mathcal{L}}{\partial \phi^{, \nu}}-g_{\mu \nu} \mathcal{L},
$$

where $\mathcal{L}$ is the Lagrangian of the theory related to the action as $I=\int \sqrt{-g} d^{4} x \mathcal{L}$. For static spacetimes $\left(T^{C}\right)_{\mu \nu}$ yields another conserved quantity which is the Hamiltonian of the system

$$
H=\int_{\mathcal{B}} T_{\mu \nu}^{C} \zeta^{\mu} d \sigma^{\nu} .
$$

In the canonical formulation of the theory $H$ plays the role of a generator of the evolution of the system along the Killing time. In general the tensors $T_{\mu \nu}$ and $\left(T^{C}\right)_{\mu \nu}$ do not coincide and their difference yields the Noether current

$$
J_{\mu}=\frac{2 \pi}{\kappa}\left(\left(T^{C}\right)_{\mu \nu}-T_{\mu \nu}\right) \zeta^{\nu},
$$

where $\kappa$ is the surface gravity. According to the Noether theorem, this current conserves $\left(\nabla^{\mu} J_{\mu}=0\right)$ on the equations of motion. As it follows from (A.2) and (A.5), the difference between the energy $E$ and the Hamiltonian $H$ is the Noether charge $Q$ corresponding to the current $J_{\mu}$.

The simplest example when $T_{\mu \nu}$ and $\left(T^{C}\right)_{\mu \nu}$ are different is the scalar field with $\xi \neq 0$. In this case

$$
\begin{gathered}
J_{\mu}=-\xi \frac{2 \pi}{\kappa}\left(R_{\mu \nu} \phi^{2}+g_{\mu \nu}\left(\phi^{2}\right)_{; \rho}^{, \rho}-\left(\phi^{2}\right)_{; \mu \nu}\right) \zeta^{\nu}, \\
H-E=\xi \int_{\partial \mathcal{B}} d s^{k}\left|g_{00}\right|^{1 / 2}\left[\left(\phi^{2}\right)_{, k}-\phi^{2} w_{k}\right]
\end{gathered}
$$

\footnotetext{
${ }^{11}$ The coefficient $2 \pi / \kappa$ in the definition (A.6) corresponds to the Killing vector $\zeta$ normalized so that $\zeta^{2}=-1$ at infinity.
} 
Here $d s^{k}$ is a three dimensional vector in $\mathcal{B}$ normal to the boundary $\partial \mathcal{B}$ and directed outward with respect to $\mathcal{B}$. Thus two energies differ by a surface term given on the boundary $\partial \mathcal{B}$ of the hypersurface $\mathcal{B}$. Obviously, when one considers a complete Cauchy surface the boundary term in (A.8) contains only a contribution from the spatial infinity, or from the external spatial boundaries if they are present. For a field falling off at infinity or obeying suitable conditions at the boundary, $E=H$. However, the situation is qualitatively different if the integration region in $E$ is restricted by the bifurcation surface $\Sigma$ of the Killing horizon, where the field $\phi$ can take arbitrary finite values. If the contribution from the spatial infinity or external boundary is absent only the Nether charge $Q$ on $\Sigma$ gives the contribution to the difference $H-E$

$$
\begin{gathered}
H-E=\frac{\kappa}{2 \pi} Q, \\
Q=2 \pi \xi \int_{\Sigma} \sqrt{\sigma} d^{2} \theta \phi^{2} .
\end{gathered}
$$

In an analogous way one can compute the charge $Q$ in other theories. For spin $1 / 2$ fields a trivial check shows that the energy and Hamiltonian are identical and $Q=0$. Presumably, $Q$ is non-trivial in the theories which include fields with higher spins.

\section{References}

[1] J.M. Bardeen, B. Carter, and S.W. Hawking, Commun. Math. Phys. 31 (1973) 161.

[2] C.W. Misner, K.S. Thorne, and J.A. Wheeler, Gravitation, San Francisco: Freeman, 1973.

[3] J.D. Bekenstein, Nuov. Cim. Lett. 4 (1972) 737.

[4] J.D. Bekenstein, Phys. Rev. D7 (1973) 2333.

[5] J.D. Bekenstein, Phys. Rev. D9 (1974) 3292.

[6] S.W. Hawking, Comm. Math. Phys. 43 (1975) 199.

[7] A. Strominger and C. Vafa, Phys. Lett. B379 (1996) 99.

[8] C.V. Johnson, R.R. Khuri, and R.C. Myers, Phys. Lett. 378 (1996) 78.

[9] J.M. Maldacena and A. Strominger, Phys. Rev. Lett. 77 (1996) 428.

[10] C.G. Callan and J.M. Maldacena, Nucl. Phys. B472 (1996) 591.

[11] G.T. Horowitz and A. Strominger, Phys. Rev. Lett. 77 (1996) 2368.

[12] G.T. Horowitz, Quantum States of Black Holes, preprint gr-qc/9704072. 
[13] A.W. Peet, The Bekenstein Formula and String Theory (N-Brane Theory), preprint hep-th/9712253.

[14] S. Carlip, Phys. Rev. D51 (1995) 632.

[15] A. Strominger, Black Hole Entropy from Near Horizon Microstates, preprint hepth/9712251.

[16] A. Ashtekar, J. Baez, A. Corichi, K. Krasnov, Quantum Geometry and Black Hole Entropy, preprint gr-qc/9710007.

[17] W.H. Zurek and K.S. Thorne, Phys. Rev. Lett. 54 (1985) 2171.

[18] G.'t Hooft, Nucl. Phys. B256 (1985) 727.

[19] K.S. Thorne, R.H. Price, and D.A. Macdonald, Black Holes: The Membrane Paradigm, Yale University Press 1986, New Haven and London.

[20] W. Israel, Phys. Lett. 57A (1976) 107.

[21] J.J. Bisognano and E.H. Wichmann, J. Math. Phys. 17 (1976) 303.

[22] W.G. Unruh and N. Weiss, Phys. Rev. D29 (1984) 1656.

[23] S. Takagi, Progress of Theoretical Physics Supplement 88 (1986).

[24] B.S. Kay and R.M. Wald, Phys. Rep. 207(2) (1991) 49.

[25] R. Laflamme, Nucl. Phys. B324 (1989) 233.

[26] L. Bombelli, R.K. Koul, J. Lee, and R.Sorkin, Phys. Rev. D34 (1986) 373.

[27] M. Srednicki, Phys. Rev. Lett. 71 (1993) 666.

[28] S. Mukohyama, M. Seriu and H. Kodama, Phys. Rev. D55 (1997) 7666.

[29] F. Larsen and F. Wilczek, Ann. Phys. 243 (1995) 280.

[30] E. Benedict and S.-Y. Pi, Ann. Phys. 245 (1996) 209.

[31] V. Frolov and I. Novikov, Phys. Rev. D48 (1993) 4545.

[32] J.D. Bekenstein, Do We Understand Black Hole Entropy?, 7th Marcel Grossman Meeting on General Relativity (1994) p. 39, gr-qc/9409015.

[33] D. Kabat and M.J. Strassler, Phys. Lett. B329 (1994) 46.

[34] C. Callan and F. Wilczek, Phys. Lett B333 (1994) 55. 
[35] T. Jacobson, Phys. Rev. D50 (1994) 6031.

[36] A.O. Barvinsky, V.P. Frolov, and A.I. Zelnikov, Phys. Rev. D51 (1995) 1741.

[37] V.P. Frolov, Phys. Rev. Lett. 74 (1995) 3319.

[38] L. Susskind and J. Uglum, Phys. Rev. D50 (1994) 2700.

[39] J.-G. Demers, R. Lafrance, and R.C. Myers, Phys. Rev. D52 (1995) 2245.

[40] D.V. Fursaev and S.N. Solodukhin, Phys. Lett. B365 (1996) 51.

[41] S.N. Solodukhin, Phys. Rev. D52 (1995) 7046.

[42] F. Larsen and F. Wilczek, Nucl. Phys. B458 (1996) 249.

[43] D. Kabat, S.H. Shenker, and M.J. Strassler, Phys. Rev. D52 (1995) 7027.

[44] M. Hotta, T. Kato, and K. Nagata, Class. Quantum Grav. 14 (1997) 1917.

[45] T. Jacobson, Black Hole Entropy in Induced Gravity, gr-qc/9404039.

[46] A.D. Sakharov, Sov. Phys. Doklady 12 (1968) 1040.

[47] A.D. Sakharov, Theor. Math. Phys. 23 (1976) 435.

[48] V.P. Frolov, D.V. Fursaev, and A.I. Zelnikov, Nucl. Phys. B486 (1997) 339.

[49] V.P. Frolov and D.V. Fursaev, Phys. Rev. D56 (1997) 2212.

[50] V.P. Frolov and D.V. Fursaev, Plenty of Nothing: Black Hole Entropy in Induced Gravity, hep-th/9703178.

[51] G.W. Gibbons, Phys. Lett. 60A (1977) 385.

[52] G.W. Gibbons, in Differential Geometrical Methods in Mathematical Physics II edited by K. Bleuler, H.R. Petry, and A. Reetz (Springer, New York, 1978), p. 518.

[53] G.W. Gibbons and M.J. Perry, Proc. R. Soc. Lond. A358 (1978) 467.

[54] J.S. Dowker and G. Kennedy, J. Phys. A: Math. Gen. 11 (1978) 895.

[55] J.W. York, Phys. Rev. D33 (1986) 2092.

[56] L. Landau and I. Lifshitz, Statistical physics, v. 1, Oxford; New York: Pergamon Press. 1980.

[57] B. Allen, Phys. Rev. D33 (1986) 3640. 
[58] G.W. Gibbons and S.W. Hawking, Phys. Rev. D15 (1976) 2752.

[59] S.W. Hawking, In: General Relativity: An Einstein Centenary Survey. (eds. S.W. Hawking and W. Israel), Cambridge Univ.Press, Cambridge, 1979.

[60] J.D. Brown, G.L. Comer, E.A. Martinetz, J. Malmed, B.F. Whiting and J.W. York, Class. Quantum Grav. 7 (1990) 1433.

[61] H. W. Braden, J. D. Brown, B. F. Whiting, and J. W. Jork, Phys. Rev. D42 (1990) 3376.

[62] J.S. Dowker, Class. Quantum. Grav. 1 (1984) 369.

[63] J.S. Dowker, Phys. Rev. D39 (1989) 1235.

[64] J.S. Dowker and J.P. Schofield, Phys. Rev. D38 (1988) 3327.

[65] J.S. Dowker and J.P. Schofield, Nucl. Phys. 327 (1989) 267.

[66] D.V. Fursaev, Euclidean and Canonical Formulations of Statistical Mechanics in the Presence of Killing Horizons, hep-th/9709213.

[67] N.D. Birrell and P.C.W. Davies, Quantum Fields in Curved Space, Cambridge University Press, Cambridge 1982.

[68] M.J. Duff, Nucl. Phys. 125 (1977) 334.

[69] K. Fujikawa, Phys. Rev. Lett. 44 (1980) 1733.

[70] K. Fujikawa, Phys. Rev. D23 (1981) 2262.

[71] E.S. Fradkin and G.A. Vilkoviskii, Phys. Rev. D8 (1973) 4241.

[72] S.W. Hawking, Commun. Math. Phys. 55 (1977) 133.

[73] S.P. de Alwis and N. Ohta, D52 (1995) 3529.

[74] G. Cognola, L. Vanzo and S. Zerbini, Class. Quantum Grav. 12 (1995) 1927.

[75] A.A. Bytsenko, G. Cognola, and S. Zerbini, Nucl. Phys. B458 (1996) 267.

[76] J.B. Hartle and S.W. Hawking, Phys. Rev. D13 (1976) 2188.

[77] H. Bateman and A. Erdelyi, Tables of Integral Transformations, v.1, New York, McGraw-Hill Book Company, Inc., 1954.

[78] R. Camporesi, Phys. Rep. 196 (1990) 1.

[79] A.A. Bytsenko, G. Cognola, L. Vanzo, and S. Zerbini, Phys. Rep. 266 (1996) 1. 
[80] V.P. Frolov, D.V. Fursaev and A.I. Zelnikov, Phys. Rev. D54 (1996) 2711.

[81] S. Zerbini, G. Cognola, and L. Vanzo, Phys. Rev. D54 (1996) 2699.

[82] R.B. Mann, L. Tarasov, and A. Zelnikov, Class. Quantum Grav. 9 (1992) 1487.

[83] S.N. Solodukhin, Phys. Rev. D54 (1996) 3900.

[84] A. Romeo, Class. Quantum Grav. 13 (1996) 2797.

[85] S.P. Kim, S.K. Kim, K.-W. Soh, J.H. Yee, Phys. Rev. D55 (1997) 2159.

[86] S.N. Solodukhin, Phys. Rev. D56 (1997) 4968.

[87] G. Cognola and P. Lecca, Phys. Rev. D57 (1998) 1108.

[88] Y.-G. Shen, D.-M. Chen and T.-J. Zhang, Phys. Rev. D56 (1997) 6698.

[89] N. Nakazawa and T. Fukuyama, Nucl. Phys. B252 (1985) 621.

[90] I.S. Gradshteyn and I.M. Ryzhik, Table of Integrals, Series, and Products, Academic Press, New York 1994.

[91] Yu. Gusev and A. Zelnikov, Nonlocal Effective Action at Finite Temperature in Ultrastatic Space-Times, preprint hep-th/970974.

[92] V.P. Frolov, W. Israel, and S.N. Solodukhin, Phys. Rev. D54 (1996) 2732.

[93] V.P. Frolov, D.V. Fursaev and A.I. Zelnikov, Phys. Lett. B382 (1996) 220.

[94] R.B. Mann and S.N. Solodukhin, Phys. Rev. D55 (1997) 3622.

[95] D.V. Fursaev and G. Miele, Phys. Rev. 49 (1994) 987.

[96] L. De Nardo, D.V. Fursaev and G. Miele, Class. Quantum Grav. 14 (1997) 3269.

[97] A.A. Vilenkin, Phys. Rep. 121 N5, 263 (1985).

[98] M.B. Green, J.H. Schwarz and E. Witten, Superstring Theory, vol. 2, Cambridge University Press, Cambridge, 1987.

[99] G.E. Volovik, Simulation of Quantum Field Theory and Gravity in Superfluid He-3, preprint cond-mat/9706172.

[100] J. Cheeger, J. Differential Geometry, 18 (1983) 575.

[101] H. Donnelly, Math. Ann. 224 (1976) 161.

[102] B.S. Kay and U.M. Studer, Commun. Math. Phys. 139 (1991) 103. 
[103] J.S. Dowker, J. Phys. A: Math. Gen. 10 (1977) 115.

[104] J.S. Dowker, Phys. Rev. D15 (1987) 3742.

[105] S. Deser and R. Jackiw, Comm. Math. Phys 118 (1988) 495.

[106] A. Sommerfeld, Proc. Lond. Math. Soc. 28 (1897) 417.

[107] G. Cognola, K. Kirsten and L. Vanzo, Phys. Rev. D49 (1994) 1029.

[108] D.V. Fursaev, Class. Quantum Grav. 11 (1994) 1431.

[109] D. Kabat, Nucl. Phys. B453 (1995) 281.

[110] D.V. Fursaev and G. Miele, Nucl. Phys. B484 (1997) 697.

[111] D.V. Fursaev, Phys. Lett. B334 (1994) 53.

[112] J.S. Dowker, Class. Quantum Grav. 11 (1997) L137.

[113] J.S. Dowker, Phys. Rev. D50 (1994) 6369.

[114] L. De Nardo, D.V. Fursaev and G. Miele, Class. Quantum Grav. 14 (1997) 1059.

[115] V. Moretti and D. Iellici, Phys. Rev. D55 (1997) 3552.

[116] V. Moretti and D. Iellici, Phys. Rev. D54 (1996) 7459.

[117] V. Moretti, J. Math. Phys. 38 (1997) 2922.

[118] R.B. Mann and S.N. Solodukhin, Phys. Rev. D54 (1996) 3932.

[119] I. Ichinose and Yu. Satoh, Nucl. Phys. B447 (1995) 340.

[120] Yu. Satoh, Study of Three-dimensional Quantum Black Holes, hep-th/9705209.

[121] M.-H. Lee and J.K. Kim, Phys. Rev. D54 (1996) (3904).

[122] M.-H. Lee, H.-C. Kim, and J.K. Kim, Phys. Lett. B388 (1996) 487.

[123] J. Ho, W.T. Kim, and Y.-J. Park, Class. Quantum Grav. 14 (1997) 2617.

[124] P.R. Anderson, W.A. Hiscock, and D.J. Loranz, Phys. Rev. Lett. 74 (1995) 4365.

[125] L. Vanzo, Phys. Rev. D55 (1997) 2192.

[126] S.W. Hawking, G. Horowitz, and S. Ross, Phys. Rev. D51 (1995) 4302.

[127] C. Teitelboim, Phys. Rev. D51 4315.

[128] A. Ghosh and P. Mitra, Phys. Lett. B357 (1995) 295. 
[129] G. Cognola, L. Vanzo and S. Zerbini, Phys. Rev. D52 (1995) 4548.

[130] D.V. Fursaev and S.N. Solodukhin, Phys. Rev. D52 (1995) 2133.

[131] R.M. Wald, Phys. Rev. D48 (1993) R3427.

[132] V. Iyer and R.M. Wald, Phys. Rev. D50 (1994) 846.

[133] T.A. Jacobson, G. Kang, and R.C. Myers, Phys. Rev. D49 (1994) 6587.

[134] R. Emparan, Phys. Rev. D51 (1995) 5716.

[135] J.L.F. Barbon and R. Emparan, Phys. Rev. 52 (1995) 4527.

[136] F. Belgiorno and S. Liberati, Phys. Rev. D53 (1996) 3172.

[137] F. Belgiorno and M. Martellini, Phys. Rev. D53 (1996) 7073.

[138] S.N. Solodukhin, Phys. Rev. D51 (1995) 609, ibid 618.

[139] D.V. Fursaev, Mod. Phys. Lett. A10 (1995) 649.

[140] W. Nelson, Phys. Rev. D50 (1994) 7400.

[141] J.D. Brown, Phys. Rev. D52 (1995) 7011.

[142] S.L. Adler, Rev. Modern Phys. 54 (1982) 729.

[143] Yu.V. Novozhilov and D.V. Vassilevich, Lett. Math. Phys. 21 (1991) 253.

[144] V. Moretti and D. Iellici, $\zeta$-function regularization and one-loop renormalization of field fluctuations in curved spacetimes, preprint-UTF 401, hep-th/9705077.

[145] I.D. Novikov and V.P. Frolov, Physics of Black Holes, Kluwer Academic Publishers, Dordrecht, 1989. 